\title{
Review
}

\section{Strategies for the etiological therapy of cystic fibrosis}

\author{
Luigi Maiuri $^{*, 1,2}$, Valeria Raia ${ }^{3}$ and Guido Kroemer ${ }^{\star, 4,5,6,7,7,8,9}$
}

Etiological therapies aim at repairing the underlying cause of cystic fibrosis (CF), which is the functional defect of the cystic fibrosis transmembrane conductance regulator (CFTR) protein owing to mutations in the CFTR gene. Among these, the F508del CFTR mutation accounts for more than two thirds of CF cases worldwide. Two somehow antinomic schools of thought conceive CFTR repair in a different manner. According to one vision, drugs should directly target the mutated CFTR protein to increase its plasma membrane expression (correctors) or improve its ion transport function (potentiators). An alternative strategy consists in modulating the cellular environment and proteostasis networks in which the mutated CFTR protein is synthesized, traffics to its final destination, the plasma membrane, and is turned over. We will analyze distinctive advantages and drawbacks of these strategies in terms of their scientific and clinical dimensions, and we will propose a global strategy for CF research and development based on a reconciliatory approach. Moreover, we will discuss the utility of preclinical biomarkers that may guide the personalized, patient-specific implementation of CF therapies.

Cell Death and Differentiation (2017) 24, 1825-1844; doi:10.1038/cdd.2017.126; published online 22 September 2017

Facts

- Mutation-specific therapies that aim at circumventing CFTR protein defects require different approaches for distinct classes of CFTR variants.

- The era of mutation-specific therapies truly started with the FDA and EMA approval of the CFTR potentiator Ivacaftor in patients bearing at least one copy of G551D or some other class III mutation.

- A combination of the CFTR corrector Lumacaftor and the potentiator Ivacaftor has been approved for the treatment of patients bearing the most common F508delCFTR mutation in homozygosis in spite of modest clinical benefits. Thus, new CFTR correctors are being tested in combination with Ivacaftor or new CFTR potentiators.

- More recent evidences indicate that CFTR operates in a context-specific dynamic network of interactor proteins, the 'CFTR functional landscape', that integrates both proteostasis and the 'social' CFTR network. Proteostasis regulators have emerged as alternative approaches to circumvent CFTR defect by improving defective proteostasis thus avoiding unwanted protein-protein interactions and reinstating desirable interactions for misfolded CFTR variants.

\section{Open Questions}

- The clinical utility of Ivacaftor is limited to a small fraction of $\sim 10 \%$ of CF patients, meaning that the largest segment of the CF population still awaits an etiological cure.

- Currently, no treatments are available for patients bearing the F508del-CFTR mutation in combined heterozygosis with another severe CFTR mutation.

- Accumulating evidence clearly indicates that targeting major systems of cellular proteostasis, can improve traffic and PM stability of F508del-CFTR. Will a variable combination of CFTR modulators (CFTR-centric approach) and proteostasis regulators configure the authentic etiological CF therapy that can be adapted to each individual patient's needs?

- Heterogeneity in the therapeutic response rate characterizes CF and makes the interpretation of clinical trials problematic. CF patients bearing exactly the same CFTR genotype may differ in their clinical response to experimental treatments. Is it the time for personlized approaches to CF therapy?

- Testing the individual responsiveness to candidate drugs requires objective and measurable indicators of drug efficacy that can be capable of either predicting or early monitoring drug efficacy. Are reliable, feasible, reproducible

\footnotetext{
${ }^{1}$ Department of Health Sciences, University of Eastern Piedmont, Novara 28100, Italy; ${ }^{2}$ European Institute for Research in Cystic Fibrosis, Division of Genetics and Cell Biology, San Raffaele Scientific Institute, Milan 20132, Italy; ${ }^{3}$ Regional Cystic Fibrosis Center, Pediatric Unit, Department of Translational Medical Sciences, Federico II University Naples 80131, Italy; ${ }^{4}$ Equipe11 labellisée Ligue Nationale Contrele Cancer, Centre de Recherche des Cordeliers, Paris, France; ${ }^{5}$ INSERM U1138, Centre de Recherche des Cordeliers, Paris, France; ${ }^{6}$ Université Paris Descartes, Paris, France; ${ }^{7}$ Metabolomics and Cell Biology Platforms, Institut Gustave Roussy, Villejuif, France; ${ }^{8}$ Pôle de Biologie, Hôpital Européen Georges Pompidou, AP-HP, Paris, France and ${ }^{9}$ Karolinska Institute, Department of Women's and Children's Health, Karolinska University Hospital, Stockholm 17176, Sweden

*Corresponding author: L Maiuri, European Institute for Research in Cystic Fibrosis, Division of Genetics and Cell Biology, San Raffaele Scientific Institute, Milan 20132, Italy. Tel: +393 311313 941; Fax: +390226434328, E-mail: maiuri.luigi @ hsr.it

or G Kroemer, Equipe 11 labelisée par la Ligue Nationale Contre le Cancer, INSERM U1138, Centre de Recherche des Cordeliers, Paris 75006, France. Tel: +33014427 76 67; Fax: +3301 442776 74; E-mail: kroemer@orange.fr

Received 27.3.17; revised 22.6.17; accepted 23.6.17; Edited by M Piacentini; published online 22.9.17
} 
and clinically/biologically relevant biomarkers of clinical efficacy available?

Cystic Fibrosis (CF) (OMIM 219700) is an autosomal recessive disease occurring in approximately 1 in 3500 live births in the Caucasian population, and, though to a lesser extent, in almost all other ethnic groups. CF results from loss of function mutations in the gene coding for the cystic fibrosis transmembrane conductance regulator (CFTR), a 1480amino acid protein functioning as a cAMP-regulated anion channel at the plasma membrane (PM) of epithelial cells and other cell types. ${ }^{1}$ The CFTR defect results in reduced epithelial chloride transport and bicarbonate secretion coupled to a wide clinical spectrum of highly heterogeneous disease manifestations. ${ }^{2}$ The CF phenotype comprises dysfunction of the exocrine pancreas, gastrointestinal disease, hepatobiliary failure, male infertility and, as a diagnostic marker, increased electrolytes in sweat. However, the most prevalent CF manifestation is a chronic progressive lung disease resulting from accumulation of viscous mucus, chronic inflammation, and persistent bacterial infections, mainly by Pseudomonas aeruginosa (PA), Staphylococcus aureus and Burkholderia cepacia $(\mathrm{BC}){ }^{2}$ This eventually culminates in respiratory insufficiency and premature death. ${ }^{3}$
Although CF is considered as a prototypic monogenetic disease, its genetics is complicated by the fact that more than 2000 different genetic variants affect CFTR (Clinical and Functional Translation of CFTR, 'CFTR2', http://www.cftr2.org/) producing variable phenotypes. ${ }^{2}$ Moreover, even patients with the same genotype exhibit clinical heterogeneity, which may result from environmental factors and modifier genes. ${ }^{2}$ Ever refined genotyping methods coupled to the availability of extensive databases has improved CF diagnostic, as well as the identification of novel genotype-based therapeutic tools (theratyping). Nonetheless, correction of the primary CFTR defect is currently partially achieved and hence is far away from replacing symptomatic therapies that already have extended the median life expectancy of CF patients beyond 40 years $^{3}$ (Box 1, Table 1).

\section{Molecular Bases of the CFTR Defect}

CFTR is a unique member of the ATP-binding cassette (ABC) transporters family that acts as an ATP-gated low conductance chloride-selective channel. ${ }^{1}$ The CFTR protein is composed by two membrane spanning domains (MSD 1 and 2), consisting of 6 membrane spanning $a$-helices (M1 to M6) and two cytosolic loops (ICLIs), two nucleotide-binding domains (NBD1 and NBD2) and one regulatory domain (RD). ${ }^{4} \mathrm{RD}$

Box 1 Emerging symptomatic therapies

Symptomatic therapies of CF include repeated courses of systemic and topical antibiotics, mucolytics (e.g., dornase-alpha), airway hydrators (e.g., hypertonic saline, mannitol), anti-inflammatory drugs, mechanical airway clearance techniques, pancreatic replacement enzymes, and supplementation of lipophilic vitamins. ${ }^{111}$ Recently cysteamine, a repurposed drug $^{35,36}$ that can be administered orally or by inhalation, has been shown to possess mucoactive, antibiofilm and antibacterial properties against Pseudomonas aeruginosa and biofilms formed by Burkholderia cepacia. ${ }^{109}$

Oral, intravenous or inhaled antibiotics are used to control acute exacerbations and chronic infection as well to prevent recurrent bacterial lung infections. ${ }^{111}$ A phase 2 , multicenter, randomized controlled study is assessing the efficacy of intravenous gallium (which has activity against $P$. aeruginosa) on adults with CF (https://www.cff.org/Our-Research/ClinicalTrials/Clinical-Trials-Happening-Now/Current and upcoming CFFT approved clinical trials 2015; https://www.cff.org/OurResearch/Clinical-Trials/Clinical-Trials-Happening-Now/, 2015). New aerosolized antibiotics ${ }^{112}$ and faster dry powder antibiotic formulations ${ }^{113,114}$ have been tested in several clinical trials. New appealing antibiotics as liposomal amikacin, ${ }^{115}$ inhaled quinolones ${ }^{116}$ and fosfomycin/tobramycin combination ${ }^{117}$ are being evaluated in clinical trials. De novo engineered cationic antimicrobial peptides aim at preventing biofilm formation by Pseudomonas aeruginosa in chronic infection. ${ }^{118}$

Since defective CFTR anion channel activity causes abnormalities in airway surface liquid (ASL), aerosolized mucolytics ${ }^{119}$ and osmotic agents ${ }^{120}$ are used to treat CF pulmonary disease. ${ }^{121,122}$ In addition, compounds that reduce hyperactivation of the epithelial sodium channel (ENaC, which mediates $\mathrm{Na}^{+}$absorption) ${ }^{123}$ thus correcting abnormal ASL and reducing mucus thickness are in development. A new chemical ENaC inhibitor (P-1037) is in phase 2 (ClinicalTrials.gov, https://clinicaltrials. gov/ct2/show/NCT02343445; 2015; Table 1). Similarly, an ENaC-targeting oligonucleotide encapsulated in lipid nanoparticles (GSK2225745) is in development. ${ }^{124}$ To restore water and electrolyte balance in CF airways, the calciumactivated chloride channel (CaCC) anoctamin 1 (ANO-1, also known as TMEM16A) and the essential component of the outwardly rectifying chloride channel ANO-6 (TMEM16F) are also being targeted. ${ }^{125}$ Another therapeutic option is the correction of the acidic $\mathrm{pH}^{126}$ in CF airways by means of aerosolized bicarbonate or other buffering agents such as tromethamine. Recently, tromethamine alone or in combination with hypertonic saline $(7 \% \mathrm{NaCl})$ has been proven to enhance bacterial killing in CF sputum. ${ }^{127}$

Other symptomatic treatments include dietary recommendations, supplementation of lipophilic vitamins and enzymes that counteract lipid malabsorption. 
Table 1 Conventional and emerging symptomatic therapies

\begin{tabular}{|c|c|c|c|c|}
\hline \multicolumn{5}{|c|}{ Restoring airway surface liquid (ASL) } \\
\hline & Mechanism of action & Preclinical Phase I Phase II Phase III Approved & Current status & Ref \\
\hline $\begin{array}{ll}\begin{array}{l}\text { Hypertonic } \\
\text { solution }\end{array} & \text { saline } \\
\end{array}$ & \multirow{2}{*}{$\begin{array}{l}\text { Improving airway } \\
\text { hydration } \\
\text { Modulating osmolarity of } \\
\text { ASL }\end{array}$} & \multirow{2}{*}{$\square$} & $\begin{array}{l}\text { Role } \\
\text { established }\end{array}$ & 119 \\
\hline Inhaled Mannitol & & & $\begin{array}{l}\text { Under } \\
\text { investigation }\end{array}$ & 120 \\
\hline \multicolumn{5}{|c|}{ Controling mucociliary clearance } \\
\hline Dornase alfa & $\begin{array}{lr}\text { Decreasing } & \text { sputum } \\
\text { viscosity } & \text { cleaving } \\
\text { extracellular DNA } & \end{array}$ & $\square$ & $\begin{array}{l}\text { Role } \\
\text { established }\end{array}$ & 113 \\
\hline $\mathrm{N}$-acetyl-- Cysteine & $\begin{array}{l}\text { Mucolytic } \\
\text { Increasing intracellular } \\
\text { antioxidant glutathione }\end{array}$ & $\square$ & No consensus & 163 \\
\hline PRX-110 & 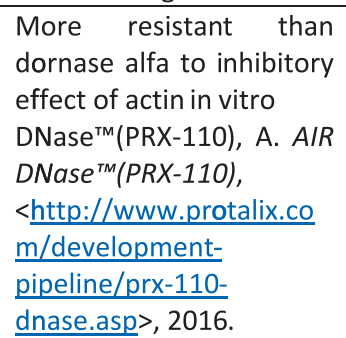 & $\square$ & & \\
\hline Gelsolin/Polyanions & $\begin{array}{l}\text { Depolymerizing } \\
\text { filamentous actin }\end{array}$ & $\square$ & & 122 \\
\hline ENaC inhibitors & $\begin{array}{l}\text { Improving mucociliary } \\
\text { clearance and targeting } \\
\text { non-CFTR channels }\end{array}$ & & & \\
\hline Amiloride & Short-lived ENaC blocker & $\square$ & No consensus & 123 \\
\hline P-1037 & $\begin{array}{l}\text { ENaC inhibitor } \\
\text { (ClinicalTrials.gov, } \underline{\text { https:/ }} \\
\text { /clinicaltrials.gov/ct2/sho } \\
\underline{w / N C T 02343445 ; 2015)}\end{array}$ & $\square$ & Ongoing & \\
\hline GSK2225745 & $\begin{array}{l}\text { Silencing } \mathrm{ENaC} \text { expression } \\
\text { with interfering RNAs- } \\
\text { siRNA }\end{array}$ & & $\begin{array}{l}\text { Under } \\
\text { investigation }\end{array}$ & 124 \\
\hline Denufosol & $\begin{array}{l}\mathrm{ENaC} \text { inhibitor through } \\
\text { stimulation of } \mathrm{CaCC}^{* *}\end{array}$ & $\longrightarrow$ & No benefit & 164 \\
\hline $\begin{array}{l}\text { SPLUNC1(short palate } \\
\text { lung and nasal } \\
\text { epithelial clone } 1 \text { ) - } \\
\text { derived peptide }\end{array}$ & ENaC inhibitor & $\square$ & & 165 \\
\hline $\begin{array}{l}\text { TMEM16A (ANO-1) } \\
\text { TMEM16F (ANO-6) }\end{array}$ & CaCC activator & & $\begin{array}{l}\text { Under } \\
\text { investigation }\end{array}$ & 125 \\
\hline
\end{tabular}


Table 1 (Continued)

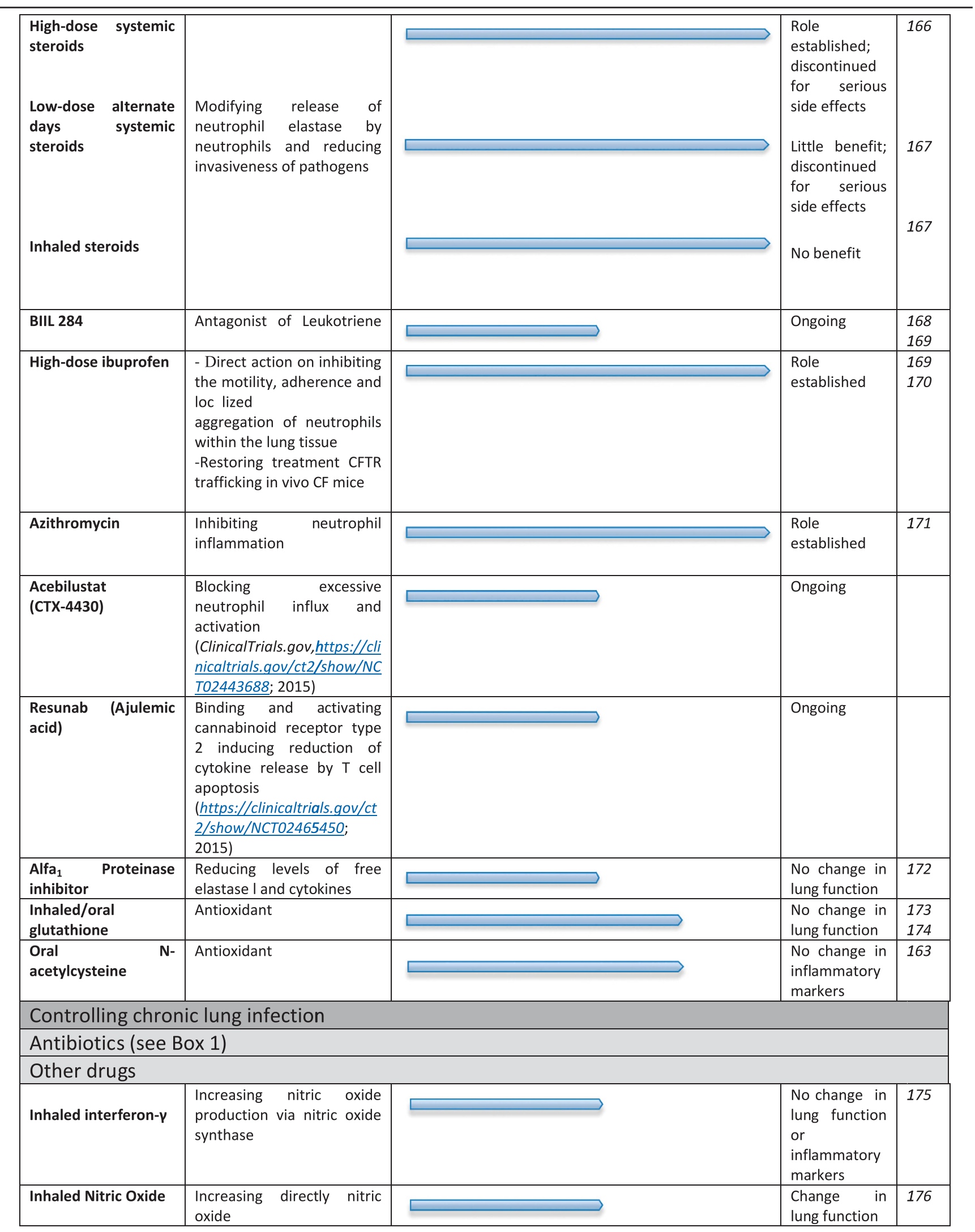


Table 1 (Continued)

\begin{tabular}{|c|c|c|c|c|}
\hline Inhaled L-arginine & $\begin{array}{l}\text { Substrate for nitric oxide } \\
\text { synthase }\end{array}$ & $\longrightarrow$ & $\begin{array}{l}\text { Change in } \\
\text { lung function; } \\
\text { no change of } \\
\text { inflammatory } \\
\text { markers }\end{array}$ & 177 \\
\hline $\begin{array}{l}\text { Inhaled } \\
\text { bacteriophages }\end{array}$ & $\begin{array}{lll}\text { In vitro effects on } \\
\text { multiresistant bacteria }\end{array}$ & $\square$ & & $\begin{array}{l}178 \\
179\end{array}$ \\
\hline $\begin{array}{l}\text { Inhaled liposomal } \beta \\
\text { glycan and OligoG } \\
\text { (saccharides) }\end{array}$ & $\begin{array}{l}\text { Disruption of Pseudomonas } \\
\text { aeruginosa biofilm } \\
\text { (ClinicalTrials.gov, } \text { https://cli } \\
\text { nicaltrials.gov/ct2/show/NC } \\
\text { T01465529; 2011) }\end{array}$ & $\square$ & $\begin{array}{l}\text { No data } \\
\text { available }\end{array}$ & \\
\hline
\end{tabular}

${ }^{a} \mathrm{CaCC}$, Non-CFTR-dependent $\mathrm{Ca}^{2+}$-activated chloride channels

contains multiple phosphorylation sites $^{5}$ that regulate the lowconductance $\mathrm{Cl}^{-}$selective channel gating at the NBDs by cycles of ATP binding and hydrolysis., ${ }^{1,6}$ ATP binding to the NBDs initiates a sequence of conformational changes that leads to NBD dimerization and channel opening. ${ }^{7}$ Since crystal structures are available for the isolated NBD1 and NBD2 only but not for the full-length CFTR, homology models have been designed. ${ }^{7-9}$ Both NBDs have two sites for ATP binding at the dimer interface. The first one (termed site 1), which tightly binds ATP, is characterized by Walker A and B motifs in NBD1 and by an LSGGQ motif (LSHGH) in NBD2, while the other (site 2), which hydrolyzes ATP, is identified by a Walker $A$ and $B$ motifs in NBD2 and by an LSGGQ motif in NBD1 (ref. 4). Molecular models predict inter-domain interactions through MSDs, ICLs and NBDs, in particular ICL2/NBD2 and ICL4/NBD1. Moreover, the 4th extracellular loop (ECL) in CFTR is glycosylated in the fully mature and folded protein. Altogether it appears that CFTR stability and function involves multiple domains of the protein, thus explaining why so many mutations affecting distinct domains of the protein may cause CF.

Classification of CFTR mutations. Approximately 2000 sequence variants affect CFTR including missense, frameshift and nonsense mutations, as well as truncations, in-frame deletions and insertions. Most CFTR mutations are very rare except the deletion of phenylalanine 508 (Phe-508del or F508del), which is present in approximately $85 \%$ of CF patients worldwide (though with some local variations), $\sim 50 \%$ of whom bear two copies of the F508del allele, while the remaining patients bear one F508del allele combined with another loss-of-function mutation (http://www. genet.sickkids.on.ca/cftr/StatisticsPage.html).

Distinct CFTR mutations can impair the synthesis, processing, PM stability, recycling or gating of CFTR protein with variable phenotypic consequences depending on the amount of residual functional protein. ${ }^{2,10}$ This led to the classification of CFTR mutations in 6 groups $^{10,11}$ with distinct functional consequences (Figure 1).

From CFTR mutations to CFTR biology. The classification of CFTR mutations stimulated the search for drugs that correct the defective function of specific CFTR mutants. However, it turned out that most mutant proteins manifest multiple (rather than simple) molecular defects and hence should have been included in more than just one class of mutations. Thus, the class II mutation F508del CFTR, also manifests hallmarks of class III mutants (a channel gating defect) and class IV mutants (increased turnover in the postER compartment, defective recycling and decreased PM stability). ${ }^{12-14}$ Similarly, the class I PTC W1282X mutant exhibits compromised NBD1-NBD2 dimerization due to a partial deletion of NBD2, leading to folding defects and reduced activity (which would be proper of class II and III mutants). ${ }^{15,16}$ To palliate the intrinsic deficiency of the initial classification of CFTR mutation in classes I-VI, an expanded classification of CFTR mutations has recently been proposed. Such a refined classification includes 31 possible classes of CFTR mutations and may support the rationale for combining several drugs that target different steps of CFTR biology. ${ }^{17}$

From CFTR mutations to CF pathology. CFTR operates in a context-specific dynamic network of interactor proteins. ${ }^{18,19}$ This CFTR network is connected to, and influenced by, the proteostasis network. ${ }^{18}$ Accordingly, the WT and F508del CFTR interactomes are rather different ${ }^{20,21}$ and fluctuations in the cellular environment (such as low temperature) modify the interaction of CFTR with other cellular proteins. ${ }^{20,21}$ These pieces of evidence support the emerging vision that CFTR does not act as a pure ion channel but that it is a major orchestrator of proteostasis ${ }^{21-23}$ Thus, defective or mutant CFTR deeply perturbs cellular homeostasis well beyond the mere absence of its chloride channel function. The dynamic network of CFTR interacting components has recently been referred to by Balch and Amaral as the 'CFTR functional landscape' (CFFL), to integrate both proteostasis and the 'social' CFTR network (SN). ${ }^{21}$ Drugs may advantageously affect the CFFL by avoiding unwanted protein-protein interactions and reinstating desirable interactions for misfolded CFTR variants, thereby improving defective proteostasis.

The pathogenesis of $\mathrm{CF}$ is connected to alterations in major cellular functions, including autophagy, ${ }^{24-30}$ the post- 
b Classes of CFTR mutations

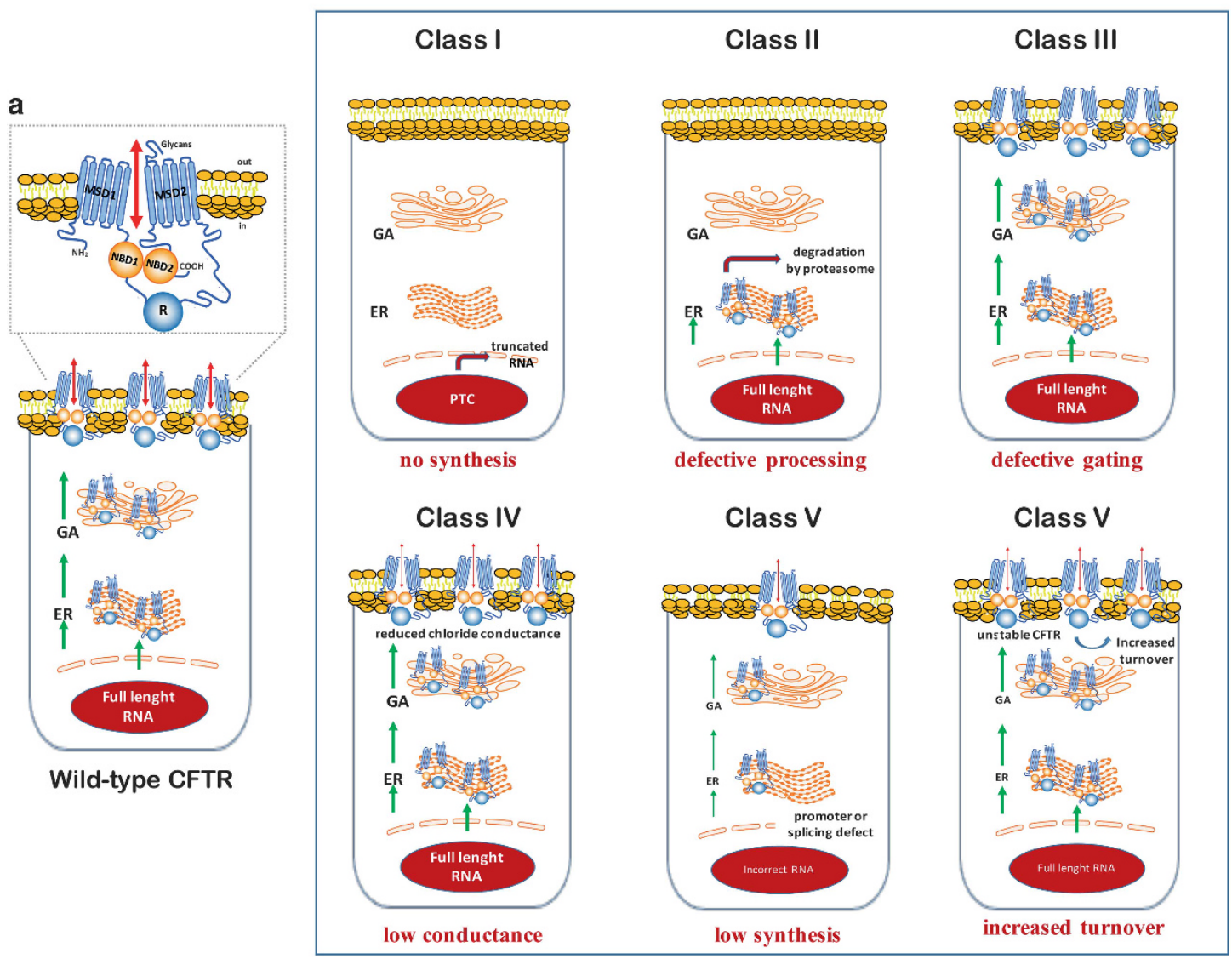

Figure 1 Classes of CFTR gene mutations. (a) Epithelial cells bearing wild-type CFTR. CFTR traffics from the endoplasmic reticulum (ER) to the Golgi apparatus (GA) and finally to the plasma membrane. (b) Impact of different classes of CFTR mutations (numbered 1-6) on synthesis, trafficking and function of CFTR. Class I mutations include nonsense, frameshift and splicing variants that prevent CFTR biosynthesis by creating in-frame stop signals (premature termination codons, PTCs) that generate a mechanism of mRNA surveillance, the nonsense-mediated decay. This causes premature arrest of translation and accelerated mRNA decay, thus reducing or abolishing the production of CFTR protein. The most common class I mutations are G542X in Mediterranean countries, R1162X and W1282X in Ashkenazy Jews. Class II mutations cause retention of misfolded proteins at the ER by local quality control mechanism. This is followed by premature ubiquitination and degradation of the misfolded protein, hence preventing its trafficking to the $\mathrm{PM}^{2,12}$ F508del affects the region of NBD1 crucial for the interdomain interaction with ICL4 of MSD2, thereby causing changes in the loop configuration between residues 509 511 with consequent topography changes of NBD1 surface. ${ }^{8,12}$ Small amounts of F508del-CFTR can reach the PM, where the CFTR mutant protein manifests poor stability and defective gating. ${ }^{12,13}$ Class III mutations are compatible with the synthesis and trafficking of CFTR mutants to the PM, but impair conduction and permeation properties of the channel. G551D, the third most common CFTR mutation accounting for 4-5\% of CFTR mutations worldwide, is located in NBD1 at the ATP-binding site 2, thus affecting ATP binding and hydrolysis, and mostly the conformational changes subsequent to ATP binding that favour channel gating. ${ }^{4}$ In spite of some differences, both G551D and G1349D, a mirror image of G551D at ATP-binding site 1, decrease the duration of channel openings and hence prolong closure periods. Class IV mutations, located mostly in the MSDs, including R117H in MSD1 [M2] and R334W or R347P in MSD1 [M6], perturb anion flow through reducing the single-channel conductance of CFTR. In these mutants, the cAMPstimulated $\mathrm{Cl}^{-}$current is greatly reduced as compared to wild-type CFTR (up to $70 \%$ for R334W) by perturbing ion-ion interaction whit in the CFTR pore. ${ }^{4}$ Class V mutations reduce protein synthesis. They include mutations that promote alternative splicing with generation of aberrant mRNA transcripts and reduced amounts of normal mRNA transcripts, as well as mutations in the promoter that reduce gene transcription. ${ }^{4}$ Class VI mutations destabilize CFTR at the PM and compromise its half-life, either by favoring CFTR disposal by endocytosis or by impairing its recycling to the PM. ${ }^{4}$ An additional class of mutations causing large deletions, named class VII, ${ }^{63}$ has been proposed to categorize untreatable mutations

translational network, ${ }^{31,32}$ and the phosphoproteome, ${ }^{33}$ all of which are influenced by, and impact on, CFTR dysfunction, often in a series of vicious cycles. ${ }^{24-26}$ Since these cellular functions can impact on F508del-CFTR trafficking, degradation and stability, ${ }^{34}$ they may represent potential targets for F508del-CFTR repair, ${ }^{21,25,29,35,36}$ as we will discuss below.

Gene therapy for CFTR repair. As a monogenic disease, $\mathrm{CF}$ is an obvious candidate for gene therapeutic approaches. Major efforts have focused on the most appropriate way to deliver a functional CFTR gene into respiratory epithelia. ${ }^{37}$
This task is rendered difficult by innate airway barriers against the entry of viral or non-viral vectors, particularly in CF patients, in which excessive mucus and inflammation may pose extra problems. Following unsuccessful attempts with adeno-associated viruses or retroviruses, the UK consortium for gene therapy promoted the optimization of plasmid-DNAliposomes. Monthly administration of this latter therapy for 1 year led to a significant, although modest improvement of lung function. ${ }^{38}$ More recently $\mathrm{F} / \mathrm{HN}$-pseudotyped lentiviral vectors have been proposed as a promising tool for clinical trials. $^{39}$ 
Box 2 Multiple F508del-CFTR defects require combination of different correctors and potentiators

Due to its intrinsic conformational defect, F508del-CFTR protein is detected by the endoplasmic reticulum (ER) quality control and destroyed before its exit from the ER (Figure 2). Given the complexity of F508del-CFTR processing, it is not surprising that the repair of F508del-CFTR is much more difficult to achieve than that of other CFTR variants with pure gating defects. ${ }^{34}$ The intrinsic thermodynamic and kinetic instability of F508del-NBD1 is largely, but not completely, corrected by the simultaneous stabilization of NBD1 energetics and NBD1-MSD2 interface in genetic revertants, i.e., second-site mutations, that lead to synergistic rescue of F508del-CFTR folding and function. ${ }^{9,61,128,129}$ Single correctors may have a modest effect on F508del because they fail to target all the multiple folding defects. Thus, combinations of two or more compounds are likely to be required to fully restore the expression of F508del-CFTR. ${ }^{59}$ An aminothiazole improves the effects of VX-809 through a mechanism that requires NBD2.

The systematic investigation of possible synergies between the CFTR corrector VX-809 and some other previously identified correctors (VX-325 or Corr-4a), culture at low-temperature $\left(26-30^{\circ} \mathrm{C}\right)$ and genetic revertants, was implemented to decipher the mode of action of compounds that target distinct portions of the F508del-CFTR protein. VX-809 targets the NBD1:TMD1 interface and VX-325 the NBD1:NBD2 interaction, while Corr-4a ${ }^{57}$ likely acts through stabilizing the core-glycosylated F508del protein at the ER level. ${ }^{61}$ The corrector Corr-4a likely stabilizes the F508del-CFTR folding intermediates upon VX-809 treatment. Bali et al., ${ }^{130}$ explored the efficacy of a triple combination of VX-809, Corr-4a and VX-770, showing that the posttranslational stabilization of Band B of F508del-CFTR by Corr-4a avoids the destabilizing effect of VX-770 (ref. $51,52)$, which otherwise increases the turnover of both CFTR Bands B and C, after rescue by the cotranslationally acting VX-809. Based on in vitro and in vivo processing experiments coupled to in silico docking analysis, Okiyoneda et al. proposed that CFTR correctors may be classified in three distinct classes that can synergize among each other for optimal F508del rescue. The class-1 correctors, including VX-809, preferentially target the primary conformational defect of NBD1MSD1 (CL1) and NBD1-MSD2 (CL4) interfaces, while class-2 correctors target the resulting NBD2 misassembly. ${ }^{60}$ In addition, chemical chaperones, as glycerol or myo-inositol, but none of the tested correctors, can stabilize F508del-NBD1, thus acting as class-3-like correctors. ${ }^{60}$ In synthesis, a structure-guided combination of different classes of correctors may target the multifaceted defect of F508del-CFTR mutant. Small molecules capable of stabilizing CFTR (CFTR stabilizers; Figure 2) might be added to cocktails of correctors and potentiators for optimal rescue of F508del-CFTR.

Recently, new avenues have been explored to correct the CFTR gene defect by genome editing. DNA editing uses engineered nucleases to remove mutated segments of the gene followed by homologous recombination with the wild-type gene. This approach ensures the cleavage at the specific sites of mutant but not of normal CFTR alleles by taking advantage of different techniques as the CRISP/Cas9 and specific RNA guides. ${ }^{40}$ RNA editing is another approach in which singlestrained antisense RNA-based oligonucleotides replace deleted mRNA segments, thus leading to the translation of repaired RNA into wild-type CFTR protein. ${ }^{41} \mathrm{~A}$ phase I study evaluates the most promising agent, QR-010 (ProQR Therapeutics, Leiden, The Netherlands), which is intranasally applied to CF patients that are either homozygous for the F508del mutation or compound heterozygous (NCT02532764).

\section{Pharmacological Strategies for CFTR Repair}

Restoring CFTR function to approximately $20-30 \%$ of the normal value is believed to confer tangible clinical benefits. ${ }^{2}$ Thus, drug discovery programs have focused on compounds capable of restoring a sufficient amount of functional CFTR protein to attenuate the CF phenotype.

Ribosomal read-through of PTCs. PTCs hinder the translation of mRNA into CFTR protein. Aminoglycoside antibiotics, as gentamicin, or the synthetic aminoglycoside derivative NB124 can promote the ribosomal 'read-through' of PTCs of class I-mutated CFTR mRNA as they favor an amino acid insertion at the PTC. ${ }^{42}$ The potential toxicity of aminoglycosides encouraged high-throughput screening (HTS) programs leading to the discovery of the orally available compound PTC124 (Ataluren), which is able to read-through PTCs but not native stop codons. PTC124 was able to promote dystrophin production in a mouse model of Duchenne's disease and CFTR expression, in mice bearing the G542X CFTR mutant. ${ }^{43}$ In addition, induction of the unfolded protein response could be an alternative strategy to stabilize transcripts with PTC for read-through by attenuating nonsense-mediated RNA decay of PTC variants. ${ }^{44}$ To date, the European Medicine Agency (EMA) granted conditional approval for Ataluren in patients with Duchenne's disease caused by PTC. Clinical trials evaluated the potential efficacy of Ataluren in CF, as we will discuss below.

Compounds targeting CFTR ion channel function: CFTR potentiators. Cell-based HTS led to the identification of small molecules capable of increasing the flow of ions through surface-localized CFTR mutants with gating defects (class III). ${ }^{45}$ Beyond the laboratory reagent VX-532, VX-770 (Ivacaftor, Kalydeco, Vertex Pharmaceutical) was the first potentiator of channel gating that obtained Food and Drug Administration (FDA) and EMA approval for the treatment of CF patients bearing the G551D-CFTR mutation, ${ }^{46}$ other specific non G551D gating mutations including G178R, S549N, S549R, G551S, G1244E, S1251N and G1349D 
and for adults with the $\mathrm{R} 117 \mathrm{H}$ mutation. ${ }^{47}$ The mechanisms through which Ivacaftor increases channel gating are still unclear, as it has similar in vitro effects on CFTR mutants affecting rather distinct domains including the signature motif of NBD1 (G551D), M2 of MSD (R117H), RD (R668C) and ICL4 (A1067T). ${ }^{16}$ Ivacaftor stabilizes the open state of CFTR, thus increasing chloride transport of wild-type CFTR and stimulates the activity of G551D-CFTR to $50 \%$ of that of non-mutated CFTR. ${ }^{45}$ Moreover, Ivacaftor enhances phosphorylation-dependent but ATP-independent channel gating by G551D CFTR and uncouples gating from ATP hydrolysis. ${ }^{48-50}$
Beyond its effects on channel gating, two research groups reported unpredicted effects for Ivacaftor. ${ }^{51,52}$ Chronic exposure in vitro to Ivacaftor destabilizes the hyper-stable G551DCFTR protein, which normally is too rigid for channel gating, hence improving its function at the PM. This concentrationdependent destabilizing effect of Ivacaftor also affects other CFTR variants that are normostable (such as wild-type CFTR) or constitutively hypostable, such as F508del ${ }^{51}$ for which Ivacaftor appears to be detrimental. This may explain, at least in part, the marginal clinical benefit of a combinatory treatment with Ivacaftor and the CFTR corrector VX-809 (Lumacaftor) (Orkambi, Vertex) that was recently FDA- and
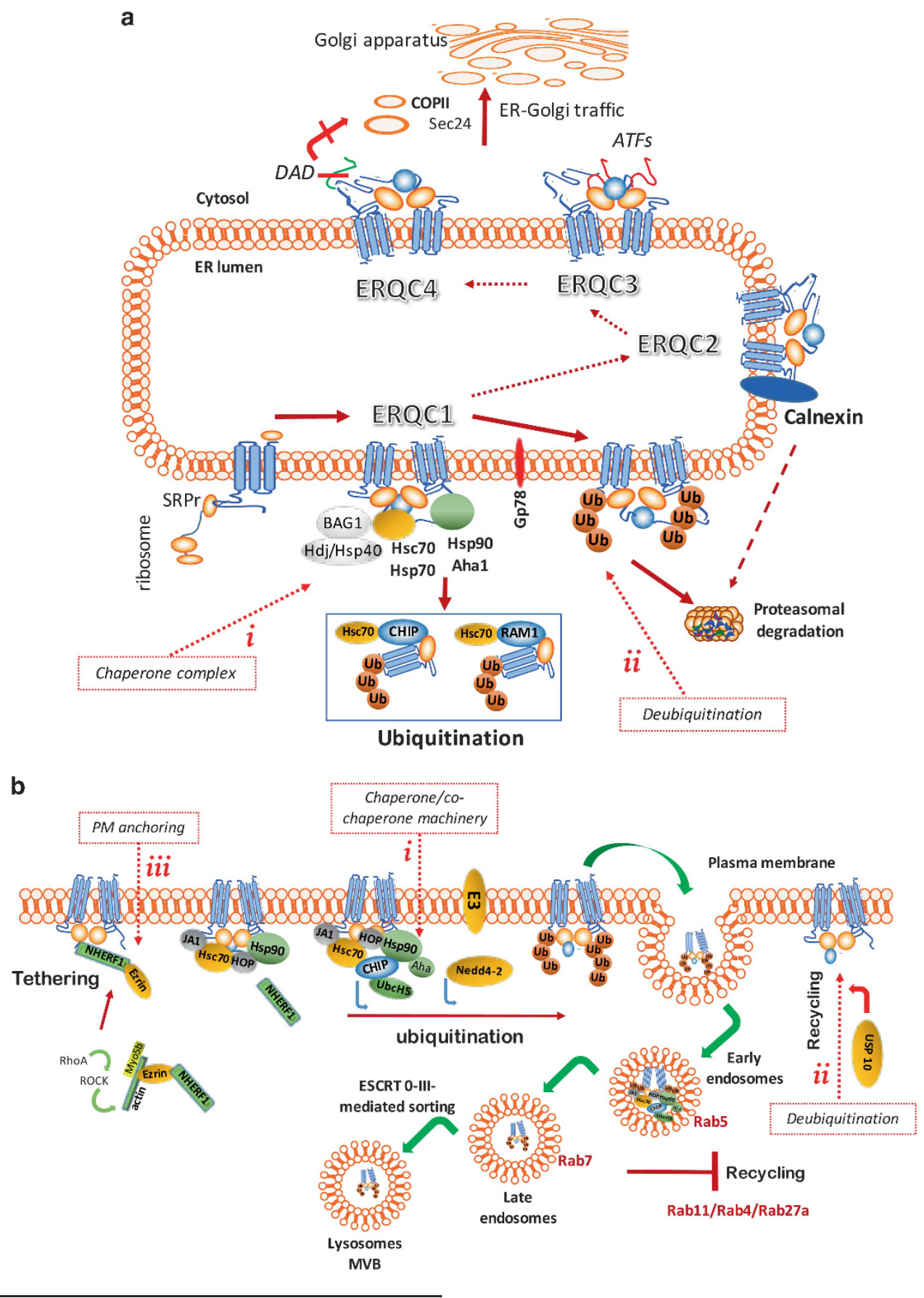
EMA- approved for the treatment of F508del-CFTR homozygous patients. ${ }^{10,53,54}$ More recently, cell-based HTS has identified several classes of potentiators (including tetrahydrobenzothiophenesthio-oxoaminothiazoles and pyrazolepyrrole-isoxazole) that, at difference with Ivacaftor, do not reduce the efficacy of F508-del-CFTR correctors ${ }^{55}$ and also weakly activate WT and G551D CFTR. ${ }^{55}$ Recently, a synergistic effect of two distinct CFTR potentiators, VX-770 and a nitro-phenylpropylamino benzoate, has been reported. ${ }^{56}$ Newly developed CFTR potentiators, as GLPG1837 (Galapagos, Mechelen, Belgium), Q8W251 (Novartis, Basel, Switzerland), FDL169 (Flatley Discovery Laboratory LCC, Charlestown, MA, USA), C-10355 and C-10358 (Concert, Lexington, MA, USA) are currently evaluated in clinical trials.

Compounds targeting CFTR protein trafficking: CFTR correctors. The F508del-CFTR mutant is a misfolded thermo-instable protein that undergoes premature intracellular degradation and can be rescued at the cell surface in vitro by culture of cells at low $\left(26-30^{\circ} \mathrm{C}\right)$ temperature. HTS led to the identification of small molecules including VX-325 (C3) and corr-4a (C4) that rescue the folding defect of F508del-CFTR (correctors) ${ }^{57}$ (Box 2). Other correctors have been identified by HTS screening and computational approaches. ${ }^{58}$ HTS performed by Vertex Pharmaceutical with the support of the Cystic Fibrosis Foundation Therapeutics, identified the correctors VX-809 and more recently, VX-661 (refs 57,59). VX-809 directly binds F508del-CFTR at the interface between NBD1 and ICL4 of MSD2 and stabilizes the NBD-MSD interface, yet it can also interact with the N-terminal fragment of CFTR, i.e., MSD1 or MSD1NBD1(refs 4,60,61). VX-809 increases the processing efficiency of F508del-CFTR and stabilizes its immature form. ${ }^{34}$ VX-809 can also improve CFTR expression in human bronchial epithelial (HBE) cells from CF donors bearing class II A561E but not class II N1303K mutations. ${ }^{62}$ However, VX-809 was less effective on HBEs bearing one copy instead of two copies of F508del-CFTR. ${ }^{62,63}$ New screening programs aim at the identification of second-generation correctors that synergize with VX-809 through binding to different sites of mutated CFTR (Box 2).

Combination treatments require heavy logistics for clinical development and may elicit unwarranted drug interactions. This prompted the search for molecules with dual (corrector and potentiator) activity. Aminoarylthiazoles (AATs) ${ }^{64}$ are an interesting example of dual active compounds. ${ }^{65}$ Moreover, 4,6,4'-trimethylangelicin was found to potentiate wild-type CFTR as well as to rescue the F508del-CFTR protein in human bronchial epithelial cells. ${ }^{66}$ In addition, CFTR modulating effects have been achieved in preclinical models by phosphodiesterase-5 inhibitors that exhibit both potentiator and corrector activity through cGMP-dependent and independent mechanisms, respectively. ${ }^{67}$ Whether these strategies may be useful for the treatment of F508del-CFTR patients, is under investigation.

\begin{abstract}
Alternative approaches to CFTR repair: proteostasis modulators. CFTR-repairing therapeutic strategies aiming at the improvement of proteostasis have emerged. ${ }^{18,21,24,25,29}$ Instead of directly targeting the mutant CFTR protein, these approaches focus on the specific cellular environment perturbed by defective CFTR function, ${ }^{18,21}$ as well as on major ER quality control (ERQC) mechanisms that may intercept mutant CFTR protein and impede its traffic to the PM (reviewed in ref. 12) (Figures $2 a$ and b). As an example, suberolyanilide hydroxamic acid (SAHA), a histone deacetylase inhibitor and regulator of heat shock protein (Hsp)90 chaperone, can rescue the function of F508del-CFTR in vitro to $28 \%$ of that of wildtype CFTR. ${ }^{68}$ Silencing the expression of the Hsp90 cochaperone Aha1, favors the exit of F508del-CFTR from the ER
\end{abstract}

Figure 2 Manipulating the cellular quality control (QC) systems to circumvent F508del-CFTR defect. (a) ERQC checkpoints. Major ERQC checkpoints test whether CFTR is eligible for ER exiting. ${ }^{12,34}$ ERQC1: chaperone trap. Nascent F508del-CFTR polypeptide chains interact the Hsp70/Hsp40 chaperone/co-chaperones that regulate, together with $\mathrm{Hsc} / \mathrm{Hdj}$-2 system, the early stages of the folding process. ${ }^{144} \mathrm{Hsp} 90$ and its co-chaperones Aha1, which strongly interacts with F508del-CFTR, and FKP8 manage later stages of CFTR folding ${ }^{69,145}$ Recruitment of the the Ub-ligases and ubiquitin adaptor proteins, as the E3 Ub ligase CHIP, E2 enzyme UbcH5, Derlin-1, E2 ligase Ubc6e or Gp78, leads to F508del ubiquitination and proteasome degradation. ${ }^{12}$ The Hsp70 co-chaperone Bag-1 can assist CFTR folding. ERQC2: calnexin cycle. Small amounts of F508del that escape ERQC1, can undergo glycan-mediated ER associated degradation (GERAD) in the chaperone/lectin cycle of calnexin which strongly binds F508del-CFTR. ${ }^{12}$ ERQC3 and ERQC4: ER exit. Either exposure of arginine-framed tripeptides (AFTs) ER-retention motifs ${ }^{146}$ or disruption of positive cargo signals (di-acidic code, DAD motif) in NBD1 compromise ER exit, Sec24-mediated packaging of CFTR into coat protein (COP) II-coated vesicles and CFTR transport to the Golgi. ${ }^{147}$ Moreover, alternative routes of CFTR transport (unconventional secretory pathways), either COPII-independent or bypassing Golgi through syntaxin 13 (ref. 148) or through GRASPs ${ }^{149}$ may deliver CFTR to the plasma membrane. Potential ERQC-targeted interventions to prevent CFTR degradation are indicated by dashed red lines and box: (i) depleting Aha1 (ref. 69); decreasing HOP by GNSOR inhibitors; ${ }^{70,71}$ overexpressing Bag-1 (ref. 150); decreasing Hsp70/CFTR interaction by Corr-4a; ${ }^{57}$ (ii) antagonizing CFTR ubiquitination by USP19 or soluble UCH-L1 (ref. 151). (b) Peripheral QC system. Additional QC mechanisms check F508del-CFTR at the PM (PQC). Chaperone/co-chaperones (including Hsc70/Hsp70/Hsp90, HOP, Hdjs, Aha1, CHIP and Bag-1), Ub-ligases and several adaptor proteins, including the assembly polypeptide-2 (AP-2), lead to the incorporation of poly-/multiple-mono ubiquitinated F508del-CFTR into clathrin-positive vesicles. ${ }^{13,152-154}$ Endocytosis. Clathrin-mediated endocytosis is facilitated by Myosins Vb/VI and Rab small GTPases, which orchestrate the fusion of early endocytic vesicles (mainly through Rab5), PM recycling (Rab4 or Rab11), sorting to late endosomes (Rab7), transport back to TGN (Rab9 and/or Rab11). Instead of being recycled to the PM, the unstable ubiquitinated F508del-CFTR is rapidly sorted to the late (Rab7 positive) endosomes and lysosomes. ${ }^{12,155} \mathrm{CFTR}$ anchoring: Up to $50 \%$ of CFTR pool is anchored to F-actin filaments ${ }^{156}$ through several PDZ proteins including NHERF-1, ezrin/radixin/moesin (ERM) and annexin 5 . $^{23,157,158}$ RhoA, Rac1 and Cdc42 GTPases modulate actin cytoskeleton reoganization and NHERF-1 binding thus regulating F508del-CFTR thetering and PM stability. ${ }^{159}$ Potential PQC-targeted interventions to stabilize F508del-CFTR and prevent PM disposal are indicated by dashed red lines and box: (i) CHIP or Aha1 ablation; ${ }^{13,69}$ (ii) USP 10 overexpression; ${ }^{151}$ (iii) NHERF-1 overexpression, which stimulates RhoA, ROCK, Rac1 signaling, ezrin phosphorylation, tight-junction organization; ${ }^{159}$ manipulation of cytocheratyn-8/F508del-CFTR association; ${ }^{160}$ modulation of Rac1 signaling through HGF, which enhances the rescuing efficacy of CFTR correctors corr-4a and VX-809 (ref. 161). Hsp, heath shock protein; Hsc, heat shock cognate protein; Aha1, Hsp90 co-chaperones activator of Hsp90 ATPase homolog 1; HOP, Hsp70/Hsp90 organizing protein; GSNOR, S-nitrosoglutathione reductase; Bag-1, Bcl-2- associated athanogenes; Ub, ubiquitin; USP, ubiquitin protease; SAHA, suberoylanilide hydroxamic acid; CHIP, Carboxy-Terminal of Hsp70 Interacting Protein; GRASP, Golgi Re-assembling and Stacking Protein; AFT, four arginine-framed RXR tripeptides; NHERF-1, Na+/H+ exchanger regulator factor isoform-1; ROCK, RhoAactivated kinase; HGF, Hepatocyte Growth Factor; ESCRT, endosomal sorting complex required for transport; MVB, multivesicular bodies 


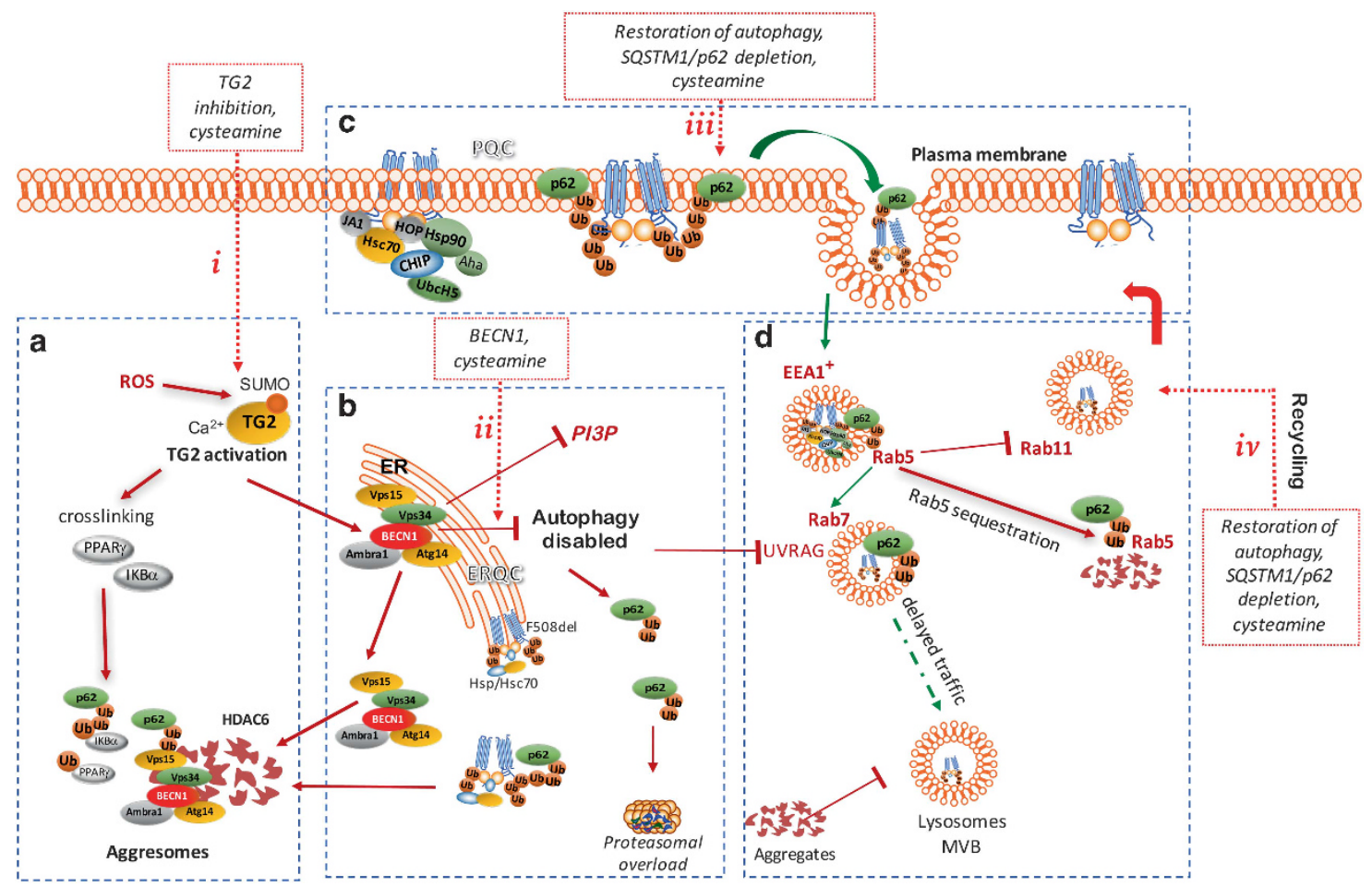

Figure 3 Defective autophagy impacts on most F508del-CFTR quality control checkpoints. (a) Defective CFTR induces ROS-mediated TG2 activation. ROS-induced PIASymediated TG2 SUMOylation sustains $\mathrm{Ca}^{2+}$-dependent TG2 activation leading to crosslinking and aggregation of substrate proteins, including PPAR $\gamma$ and IKB $\alpha^{31,32}$. Most TG2 interactor proteins are molecular chaperones which impact on CFTR processing. ${ }^{73}$ (b) TG2 activation disables autophagy. Activated TG2 crosslinks BECN1 and dislodges the phosphatidyl-inositol-3-kinase complex 3 (PI3KC3) away from the ER, thus inhibiting autophagosome formation and disabling autophagy. Defective autophagy induces accumulation of the autophagic substrate SQSTM1/p62, that targets ubiquitinated proteins, including F508del-CFTR, leading to proteasome overload and sequestration of aggregated proteins within HDAC6+/SQSTM1+ aggresomes. ${ }^{24}$ (c) Accumulation of SQSTM1/p62 at the PM favors F508del-CFTR disposal. SQSTM1/p62, a critical regulator of internalization, trafficking and sorting of ubiquitinated surface proteins, accumulates at the epithelial surface, binds PM-located ubiquitinated F508del-CFTR and colocalizes with mutant CFTR within enlarged early endosomal antigen (EEA) $1^{+}$vesicles. ${ }^{24,26}$ (d) Sequestration of PI3KC3 impairs endosomal trafficking. The sequestration of PI3KC3 reduces the abundance of phosphatidyl-inositol-3-Phosphate (Ptdlns $3 P$ ) at the EEA $1^{+}$endosomes, thus impairing endosomal fusion/maturation, and hence CFTR recycling. The PI3KC3 sequestration reduces the availability of the BECN1-interactor UVRAG that interacts with the HOP complex, thus recruiting and activating Rab7 and favoring Rab5 to Rab7 transition. ${ }^{162}$ Moreover, SQSTM1/p62 targeting reduces Rab5 levels at the EEA1 ${ }^{+}$vesicles. ${ }^{22,24}$ Defective autophagy compromises CFTR recycling through Rab11+ vesicles and diverts CFTR recycling to lysosomal degradation. Moreover, it impairs Rab5-Rab7 transition, thus delaying CFTR trafficking to the late endosomes. ${ }^{22,24,25}$ Potential targeted interventions to circumvent F508del-CFTR defect are indicated by dashed red lines and box. (i) TG2 depletion or inhibition by cysteamine or (ii) BECN1 overexpression can reestablish autophagy flux, increase PI3P availability and prevent SQSTM1/p62 accumulation, thus ultimately favouring F508del-CFTR rescue and PM stability. (iii) direct SQSTM1/ p62 depletion or the enforced expression of SQSTM1/p62 mutants lacking the UBA domain, increases F508del-CFTR PM stability and autophagy flux. ${ }^{22,24,25}$ BECN1, Beclin 1; SQSTM/p62, sequestrosome 1; ROS, reactive oxygen species; TG2, transglutaminase 2; UVRAG, UV-irradiation-resistant-associated-gene

and its traffic to the PM. ${ }^{69}$ Moreover, the modulation of Hsp70/ Hsp90 organizing protein (HOP) nitrosylation by the inhibitor of S-nitrosoglutathione reductase (GSNOR) N91115, prevents mature CFTR degradation in epithelial cell cultures. ${ }^{70,71}$ More general proteostasis modulators aim at subtly redressing the perturbed proteostasis in CF, mostly by enhancing autophagic flux ${ }^{24,25,35,36}$ (Figure 3 ) and by modulating the phosphoproteome. $^{33,35,36}$

Proteostasis modulators that stimulate autophagy: Epithelial cells bearing the F508del-CFTR mutation manifest an increased tendency of protein aggregation affecting the NBD1 domain of CFTR, ${ }^{24,72}$ as well as regulatory and anti-inflammatory proteins. ${ }^{24,31,32}$ Increased generation and defective detoxification of reactive oxygen species (ROS) entail changes of the post-translational network, including SUMOylation of the multifunctional enzyme transglutaminase-2 (TG2), ${ }^{73}$ thus preventing its ubiquitin-mediated degradation and sustaining TG2 activation ${ }^{31,32}$ (Figure 3). TG2 in turn stimulates protein aggregation. ${ }^{32}$ Most intracellular chaperones, including those involved in CFTR trafficking, interact with TG2. ${ }^{73}$ The overactive TG2 in CF epithelia sequesters several substrate proteins, ${ }^{32}$ including the master regulator of autophagy, Beclin 1 (BECN1), hence suppressing autophagy, ${ }^{24}$ a key pathway of stress-induced adaptation to cell-autonomous or environmental stresses. ${ }^{74,75}$ (Figure 3). Defective autophagy results in the accumulation of the autophagic substrate SQSTM1/p62 (ref. 76), which binds to ubiquitinated F508del-CFTR, thus favoring either its degradation and PM disposal. ${ }^{22,25}$ Inhibition of the BECN1dependent class 3 phosphatidyl-inositol-3 (Ptdlns3)-kinase (Ptdlns3K, also known as hVps34) reduces the availability of Ptdlns3-phosphate (Ptdlns3P), ${ }^{77}$ thus inhibiting endosomal trafficking and the recycling of PM proteins, including CFTR $^{22}$ (Figure 3). Apparently, CFTR, TG2 and autophagy can impact on each other in feed-forward loops. Thus, CFTR inhibition activates TG2 and inhibits autophagy, while 
conversely TG2 inhibition and autophagy induction both have a positive impact on the abundance of functional F508delCFTR protein. Notably, TG2 activation inhibits autophagy, and the TG2 interactome is remodeled upon autophagy induction. Thus, TG2 and autophagy intersect most quality control checkpoints that dictate the rules of F508del-CFTR processing, trafficking and PM stability. ${ }^{73}$

Restoring autophagy flux by genetic interventions (enforced expression of BECN1), depletion of SQSTM1/p62 or inhibiting TG2 by means of the proteostasis regulator cysteamine, can rescue F508del-CFTR expression at the PM and restore its function in human bronchial epithelial cell lines, in primary nasal cells from F508del CF patients, as well as in vivo in transgenic mice bearing the equivalent of the F508del mutation $^{24,25}$ (Figure 3). As a consequence, cysteamine reduces lung inflammation and prevents the juvenile overmortality of F508del-CFTR mice due to intestinal obstruction. $^{35}$ Cysteamine effects on PM expression of F508del-CFTR last $24 \mathrm{~h}$ beyond its washout. ${ }^{25,35}$ Moreover cysteamine effects on lung inflammation persist for several days following its withdrawal. ${ }^{35}$ These results suggest that cysteamine can both rescue and stabilize F508del-CFTR protein at the PM and that targeting autophagy may help circumventing the CFTR defect ${ }^{35,36}$ In addition, autophagy stimulation by rapamycin or SQSTM1/p62 depletion restores bacterial killing by F508del-CFTR macrophages. ${ }^{27-30}$ Recently, Romani et al. reported that the naturally occurring polypeptide thymosin $a 1$ is endowed with a dual ability to control inflammation and rectify the functional defects of F508del-CFTR through restoring autophagy, both in F508delCFTR homozygous mice and human bronchial epithelial cells. $^{78}$

Altogether these results suggest that stimulation of autophagy may improve CF caused by the frequent F508del-CFTR mutation.

Proteostasis modulators that target the phosphoproteome: CFTR channel function is deeply influenced by its phosphorylation status (reviewed in ref. 33). The open probability of CFTR channel is increased by the protein kinase A (PKA)mediated phosphorylation of the $\mathrm{R}$ domain. ${ }^{12,33}$ Other kinases can favor CFTR opening (as exemplified by cGMPdependent protein kinase SRC and prolin-rich tyrosine kinase PYK2), ${ }^{79}$ inhibit CFTR channel activity (AMP-dependent protein kinase, AMPK, and lemur tyrosine kinase, LMTK2) ${ }^{80}$ or exert a dual activity on its function (PKC). ${ }^{33}$ Beyond these direct phosphorylation reactions affecting channel function, a wide array of kinases influence CFTR traffic, stability and function. $^{33}$ Among others, a crosstalk between spleen tyrosine kinase (SYK) and the with-no-lysine (WNK) protein kinases can regulate CFTR trafficking to the PM, as well as $\mathrm{PM}$ residence. More importantly, the master kinase protein kinase CK2 (CK2) phosphorylates CFTR and regulates CFTR biogenesis at multiple levels in a hierarchical synergy with SYK. ${ }^{80}$ The interplay between CK2 and CFTR is bilateral in thus far that CK2 stimulates proteolytic CFTR degradation and the resulting CFTR fragment allosterically activates CK2, hence closing a positive feed forward loop. ${ }^{81-83}$ Accordingly, CX-4945, a selective CK2 inhibitor, stabilizes F508del-CFTR protein at the PM following rescue by cysteamine. ${ }^{35}$ The search of natural compounds that act similarly as CX-4945 led to the discovery of the over-the-counter nutraceutical epigallocatechin-gallate (EGCG) as a CK2 inhibitor. Similarly to CX-4945, EGCG stabilizes F508del-CFTR at the PM for up to $48 \mathrm{~h}$ following cysteamine washout in primary nasal epithelial cells from F508-CFTR homozygous and compound heterozygous patients. ${ }^{35}$ EGCG also prolongs the beneficial effects of cysteamine for several weeks in F508del-CFTR homozygous or F508del/null CFTR mice. ${ }^{35,36}$ Two open-label phase 2 trials in F508-CFTR homozygous and compound heterozygous patients demonstrated that sequential treatment with cysteamine and EGCG significantly decreased sweat chloride concentrations and reduced airway inflammation. ${ }^{35,36}$ Thus, combinations of distinct proteostasis regulators may be efficient against CF. Future research will establish optimal combinations and treatment schedules for such proteostasis regulators.

CFTR-centric strategies versus proteostasis modulators: two apparently antinomic views. Two major differences oppose proteostasis modulating strategies and CFTR-centric strategies (i.e., correctors and potentiators). First, proteostasis modulation is likely to have a broader action than CFTR correctors and potentiators because the latter directly interact with specific CFTR mutants. Secondly, the effects of proteostasis regulators strictly rely on the cellular mechanisms through which they indirectly rescue CFTR mutants. An interesting example is offered by cysteamine that restores a functional F508del-CFTR only in an autophagycompetent context. ${ }^{36}$ Whether correctors and potentiators remain active in conditions of suppressed autophagy has not been determined, yet may be expected from their mode of action.

These two major differences in the mode of action between proteostasis regulators and CFTR-targeting molecules, suggest that these two a priori antinomic approaches may complement one another, thus giving rise to new combination regimens.

\section{Strategies for Preclinical Validation of Candidate Drugs}

Recent clinical trials with CFTR correctors, alone or in combination with Ivacaftor, in patients bearing the F508delCFTR mutation, raise more questions than answers. The translation of investigational drugs into clinical application revealed a major difference between the promising effects observed in cultured cells and rather modest effects in patients. ${ }^{53,54}$ Phase 3 clinical studies with combined theraphy ${ }^{54}$ did not include F508del-CFTR rescue as a primary or secondary endpoint, leading to the paradoxical situation that biomarkers used to measure efficacy in cells and in patients are not the same.

Logically, preclinical models should be as close a possible to the clinical setting and feasibility should be a requirement for validation tests in large-scale screenings. ${ }^{36,84-88}$ Indeed, the higher the number of compounds to be tested, the faster the screening procedure should be (Box 3 ). In addition, translational medicine requires pre-clinical validation of drug candidates in vivo in appropriate animal models of disease ${ }^{89-93}$ (Box 3). 
Box 3 Preclinical validation of candidate drugs

\section{In vitro and ex vivo testing of candidate drugs}

Heterologous expression systems (in which the mutant CFTR gene is introduced into cancer cell lines) should progressively be replaced by patient-derived primary culture models. ${ }^{84}$ The assessment of CFTR ion channel function in Ussing chambers followed by biochemical detection of mature CFTR protein in primary long-term cultures of bronchial (HBE) or nasal scrapings from CF donors, likely constitutes the best tool to test drug candidates. ${ }^{62,85}$

Primary intestinal organoids obtained by suction rectal biopsies from CF patients might fulfill this task. Rectal organoids can be indefinitely grown and expanded as they contain stem cells, allowing a continuous source of patient-derived biological samples. In response to the cAMP-elevating compound forskolin, organoids obtained from normal subjects swell, while those from CF patients fail to do so. ${ }^{86}$ Measuring the magnitude of swelling of cultured organoids hence may be used in medium- and high-throughput screening programs. ${ }^{86}$ Nonetheless, the responses of epithelial scrapings from CF donors to in vitro treatments are afflicted by high variability ${ }^{87}$ and, if such cells have been longterm cultured in vitro, they are unlikely to reflect the 'actual' disease state of CF patients.

Short-term ex vivo cultures of freshly isolated nasal cells constitute a useful tool for predicting the responsiveness of individual patients to candidate drugs. ${ }^{35,36}$ In this model, fluorescence-based measurements of CFTR function, biochemical detection of PM resident mature CFTR, as well as the assessment of pathogenic and inflammatory biomarkers can predict drug efficacy in vivo. ${ }^{35,36}$ In addition, several reports suggest that freshly drawn blood monocytes may be used to assess the efficacy of test compounds with respect to CFTR rescue. ${ }^{88}$ It will be important to systematically compare the response of distinct cell types to pharmacological CFTR rescue to the clinical effects observed in the patients from which cells have been obtained.

\section{Pre-clinical validation of drug candidates in vivo: mouse models of cystic fibrosis}

Many transgenic mouse models of CF have been generated. However, 'CF mice' do not recapitulate the clinical manifestations of CF lung disease, ${ }^{89,131}$ likely due to major physiological and microbial differences between rodents and humans. CF mice only manifest a mild inflammatory lung phenotype. coupled to hyper-responsiveness to bacteria or microbial products ${ }^{90,91}$ that may be measured experimentally. Nevertheless, CF mice may be useful to test candidate drugs.

Murine and human CFTR exhibit a 78\% amino acid sequence homology. The first CFTR knockout (CFTR ${ }^{\text {tm } 1 U N C}$ ) mice were generated by the insertion of a stop codon in exon 10 (S489X mutation), but other knockout mice including CFTR ${ }^{\operatorname{tm} 1 C A M}, C^{2}$ CFR $^{\text {tm1BAY }}, C^{\text {CFTR }}{ }^{\text {tm3BAY }}$ and CFTR $^{\text {tm1HSC }}$ have been generated. Now, multiple knock-in mice bearing the most common CFTR mutation F508del, CFTR ${ }^{\text {tm1EUR }}$, CFTR $^{\text {tm1KTH }}$, CFTR $^{\text {tm2CAM }}$, or mutations G480C $\left(\right.$ CFTR $\left.^{\text {tm1HGU }}\right)$, G551D (CFTR ${ }^{\text {tm1G551D }}$ ), G542X are available (reviewed in ref. 89).

In contrast to patients with CF, mice carrying equivalent mutations in the CFTR gene fail to develop severe lung disease unless they are experimentally challenged. ${ }^{89,131}$ Instead, CF mice often die from intestinal obstruction, which only occurs in a minority of infants with CF. ${ }^{89}$ CF mice can manifest spontaneous inflammation and mount hyperinflammatory responses upon challenge with live bacteria or lipopolysaccharide (LPS). ${ }^{25,35,36,91,132}$ Early changes in respiratory mechanics and distal airspace enlargement have been described in C57BI/6 mice bearing F508del or R117H CFTR mutations in the absence of infections, similarly to the early lung phenotype of CF patients. ${ }^{90}$ Agarose beads containing Pseudomonas can be used to cause chronic endobronchial infections in CF mice. ${ }^{133}$ Repeated exposure of CFTR knockout mice to LPS causes abnormal and persistent immune response and inflammation associated with robust architectural changes and lung remodeling that are not observed in wild-type littermates. ${ }^{91}$

Although differences in genetic background determine variations in the phenotype ${ }^{89} \mathrm{CF}$ mice may be useful to test candidate drugs, provided that appropriate biomarkers are selected. The orally bioavailable read-through agents PTC124 and NB124, have been tested in G542X mice in which they both partially restored CFTR protein expression in the intestine. ${ }^{43}$ In mice bearing the F508del-CFTR mutation, glafenine partially restored CFTR trafficking defect. ${ }^{134}$ Moreover, a 6-day treatment with n-butyldeoxynojrimicin (Miglustat) improves sodium and chloride transport across epithelial layers. ${ }^{135}$ More recently, CFTR ${ }^{\operatorname{tm} 1 E U R}$ mice, have been used to demonstrate the ability of proteostasis modulators, ${ }^{24,25,31,35}$ to (i) rescue CFTR protein expression and function, (ii) reduce lung inflammation, and (iii) prevent the juvenile overmortality due to intestinal obstruction. ${ }^{35}$ Moreover, Tosco et $a .^{36}$ generated a new CFTR ${ }^{\mathrm{F} 508 \mathrm{del} / \text { null }}$ mouse that may be considered as a model of F508del composite heterozygous patients.

CF mice can provide information on the mechanisms through which a candidate drug rescues CFTR function. As an example, inhaled lentiviral vectors can be used to overexpress proteins (such as BECN1), or to silence proteins (such, as SQSTM1/p62) by RNA interference, with the scope of mimicking the positive effects of cysteamine on F508del-CFTR function lung inflammation. ${ }^{36}$ Tosco et al. provided genetic proof that combination therapy with cysteamine and EGCG improves CF via the induction of autophagy by backcrossing the CFTR ${ }^{\text {tm1EUR }}$ defect into a Becn1 haploinsufficient (i.e., autophagy-deficient) background, in which the combination regimen failed to restore CFTR function.

Transgene-enforced overexpression of the $\beta$ subunit of the ENaC channel ( $\beta$-ENAC mice) with consequent overactivation of the ENaC channel and $\mathrm{Na}^{+}$hyperabsorption, recapitulates the clinical CF lung phenotype more accurately than the mutation of CFTR. ${ }^{136}$ While this model can be used to dissect the pathogenesis of pulmonary disease in CF, it is not useful to test etiological CF therapies.

Recently, pig $^{92}$ and ferret ${ }^{93}$ models of CF have been developed to better mimic the human disease. In spite of their size and cost, 'CF pigs' promise to constitute a valuable tool to study CF disease mechanisms. ${ }^{92}$ 


\section{Strategies for Personalization of CF Treatments}

Even patients bearing exactly the same CFTR genotype may differ in their clinical response to experimental treatments, calling for patient-centered (rather than mutation-centered) approaches to therapy. The emerging paradigm of precision medicine posits that clinical development requires novel ways of testing interventions that focus on individual responses ( $\mathrm{N}$-of-1 clinical trials), rather than average responses of a cohort of patients. Testing the individual responsiveness to candidate drugs requires objective and measurable indicators of drug efficacy ${ }^{94}$ that support clinical management.

Intermediate clinical evaluations (like the frequency of respiratory exacerbations or the need for antibiotics) or late clinical endpoints (like performance and survival) are usually anticipated by established 'surrogate' end-points of efficacy, such as spirometric measurements including $\mathrm{FEV}_{1} \%$ predicted or the lung clearance index. ${ }^{95,96}$ However, such spirometric parameters are subjected to high inter- and intraindividual variability ${ }^{96}$ and constitute relatively late indicators of efficacy. For this reason, measurable early biochemical/ functional biomarkers are needed.

As recommended by the European Cystic Fibrosis Society (ECFS) Clinical Trial Network, biomarkers should be reliable, feasible, reproducible and clinically/biologically relevant with minimal risk/discomfort to patients. ${ }^{94}$ The ideal biomarker(s) should help trial management at two levels. First, biomarkers should predict the responsiveness of an individual patient to candidate drugs before treatment, and hence influence the decision whether to treat or not. Second, they should also monitor early treatment responses before late long-term clinical benefits become evident, and hence affect the decision whether to (dis)continue the treatment. Ideally, the same biomarker(s) should fulfill both needs (Box 4).

Futures studies should aim at the large-scale validation of the most reliable, feasible and affordable biomarker (or combination of biomarkers) that accurately predicts clinical endpoints.

\section{Clinical Evaluation: The Reality Check}

Mutation-specific therapies require different approaches for distinct classes of CFTR variants. Read-through agents are useful for patients bearing class I CFTR mutations. A phase 3 trial during which Ataluren was administered for 48 weeks revealed that Ataluren lowered the decline in lung function only in the subgroup of patients who did not receive the inhaled aminoglycoside tobramycin. ${ }^{97}$ As a result, new phase 3 trials excluding patients who inhale aminoglycosides have been launched (https://clinicaltrials.gov/show/NCT02139306,2014; Clini calTrials.gov, https://clinicaltrials.gov/ct2/show/NCT02456103, 2015; Table 2). However, it has been recently announced that these trials failed to achieve primary and secondary endpoints. $^{98}$ Thus, clinical development of Ataluren for CF has been discontinued.

The era of mutation-specific therapies truly started with the FDA and EMA approval of the CFTR potentiator Ivacaftor in patients at different ages and clinical conditions bearing at least one copy of G551D or some other class III mutation. ${ }^{46,47,99-101}$ Ivacaftor fulfills all requirements of CFTR repairing therapies as it: (i) corrects the CFTR defect in vivo (as indicated by the fact that it decreases sweat chloride below $50 \mathrm{mmol} / \mathrm{l}$, i.e., to the normal range); (ii) increases the $\mathrm{ppFEV}_{1}$ as an objective parameter of respiratory function and reduces pulmonary exacerbations; and (iii) improves nutritional status and the overall quality of life. ${ }^{102}$ Post-marketing studies confirm long-term drug-dependent clinical benefits. ${ }^{100}$ Although rather expensive, ${ }^{103}$ Ivacaftor exemplifies an etiological therapy that is useful for approximately $10 \%$ of $\mathrm{CF}$ patients. However, Lumacaftor, which efficiently corrects deficient F508del-CFTR expression in vitro ${ }^{59}$ yielded rather modest effects on sweat chloride and a negligible improvement of lung function in patients ${ }^{104} \mathrm{~A}$ combination of Lumacaftor and the potentiator Ivacaftor (Orkambi) was tested in a phase 2 study $^{53}$ and then in two double-blind placebocontrolled phase 3 clinical trials, in patients homozygous for the F508del-CFTR mutation. ${ }^{54}$ Nevertheless, the effects of the sweat test (or other direct measurements of CFTR function) were not disclosed. ${ }^{54}$ Pooled data analysis revealed a significant, though modest increase of the ppFEV 1 (2.6$4.0 \%$ ) at week 24 and a $30-40 \%$ reduction in the number of pulmonary exacerbations, as defined by the need of additional antibiotics. ${ }^{103}$ However, based on the CF QuestionnaireRevised (CFQ-R) there was no significant improvement of the quality of life. ${ }^{105}$ It can be speculated that the marginal improvement of lung function without subjective benefits and without an objective increase in CFTR function, could reflect an 'off-target' effect of the combination regimen. Indeed, symptomatic treatments with the antibiotic azithromycin or recombinant human deoxyribonuclease I (Dornase alfa) can improve ppFEV $_{1}$ (ref. 106), indicating that even agents that do not improve CFTR function may have some impact on lung function. In spite of its limitations, the combination of Lumacaftor and Ivacaftor has been approved for the treatment of F508del-CFTR homozygous patients aged $\geq 12$ years in several countries. ${ }^{10}$ Recently, a phase 3 extension study (72-96 weeks; PROGRESS) showed a slower rate of ppFEV 1 decline than in matched registry cohort. ${ }^{107}$

New CFTR correctors are being developed by several companies. Ongoing phase 2-3 clinical trials are evaluating the efficacy and safety of combination of the corrector VX-661 with Ivacaftor (ClinicalTrials.gov,https://clinicaltrials. gov/.). These studies include hundreds of patients bearing F508del-CFTR in homozygosis or in composite heterozygosis with a mutation resulting in minimal or residual CFTR function or gating defects (Table 2). New CFTR potentiators are also being tested in phase 1 and 2 a clinical trials in combination with CFTR correctors. Recently, Vertex has announced a phase 1 study to evaluate the triple combination of VX-661 with two next generation correctors, VX-152 (ClinicalTrials. gov,https://clinicaltrials.gov/show/NCT02951195; 2017) and VX-440 (Table 2).

Proteostasis regulators have emerged as an alternative strategy for F508del-CFTR repair. An orally available inhibitor of S-nitrosoglutathione reductase (GSNOR), N91115, that stabilizes F508del-CFTR through targeting the Hsp70/ Hsp90 organizing protein, ${ }^{70}$ is in phase 2 clinical trials in combination with Orkambi (NCT02589236) or with Ivacaftor (NCT02724527) in patients bearing F508del-CFTR or gating mutations, respectively (Nivalis Therapeutics). In addition, the 


\section{Box 4 Toward a personalized CF therapy}

Personalized medicine posits the adaptation of therapeutic interventions to individual patients. Indeed, population-based clinical usually lead to the discovery of patient subgroups who either are refractory to the treatment or manifest major toxicities. Ever sophisticated molecular analyses of genes, proteins, microbiomes and metabolites favor the personalization of treatments.

Besides measurements of drugs capability of restoring CFTR function in vivo, additional markers of disease reversion have been implemented to anticipate early potential clinical benefits. ${ }^{94}$

\section{Biomarkers of CFTR function}

Assessing CFTR function as early detector of the repair of CFTR defect should be mandatory for etiological therapies. CFTR function can be measured in vivo assessing the sweat chloride concentrations, the nasal potential difference (NPD) or intestinal current measurements (ICMs). As recently reported for Ivacaftor ${ }^{137}$ and proteostasis regulators, ${ }^{36}$ the sweat chloride concentration constitutes a feasible and appropriate biomarker of CFTR function in vivo, in spite of its high inter-patient variability. ${ }^{97,137}$ NPD can provide information on both chloride secretion and sodium absorption at a disease-relevant site, in spite of considerable withinsubject variability. ${ }^{97}$ NPD has been shown to possibly reveal patient's responsiveness in clinical trials. ${ }^{46,97,137}$ However, NPD needs the active cooperation by the patient, limiting its utility in infants. ICMs are performed on suction rectal biopsies in micro-Ussing chambers ${ }^{138}$ and discriminate between CF and non-CF individuals, yet are poorly acceptable to patients.

Nonetheless, none among the aforementioned tests can be used to either predict and monitor the responsiveness of patients to treatment. Prolonged cultures of nasal or bronchial epithelial cells or intestinal organoids are potentially useful to predict drug efficacy. As a drawback, these procedures require the time-consuming expansion of primary cells, meaning that the cells are unlikely to preserve the changes induced by prior drug exposure in vivo and hence cannot be used to monitor pharmacological effects. Hence, preference has to be given to freshly explanted cells that can be analyzed for predictive or pharmacodynamics biomarkers ex vivo. Epithelial cells that are freshly collected by nasal brushing and then analyzed ad hoc, without further in vitro expansion, may better fulfill this task.

\section{Mechanistic biomarkers}

Beyond the assessment of CFTR expression and function (which obviously yields the most accurate 'on target' biomarker), other biomarkers can be explored. Thus, mechanistic biomarkers (like the abundance of BECN1 and STQM1/p62 as well as the lipidation of LC3) inform whether a candidate drug induces autophagy. Moreover, the detection of early indicators of disease reversion (as the expression of inflammatory cytokines at the mRNA and protein levels) can be monitored to predict potential clinical benefit.

\section{Biomarkers of inflammation}

Broncho-alveolar lavage (BAL) allows to directly measure biomarkers of lung inflammation. However, the invasiveness of BAL collection limits its application. Induced or spontaneously expectorated sputum can replace BAL in collaborating adult and adolescents patients. ${ }^{139}$ Nasal brushings freshly collected from CF patients have been shown to recapitulate many features of lower airways and can represent a simple and feasible procedure in both young and adult patients. ${ }^{35,36}$ Exhaled breath condensate mostly consisting of condensed water vapor with a small and variable fraction of airway secretions, can be an additional non-invasive procedure to assess non-volatile compounds contained in peripheral airway surface liquid, including cytokines, nitrates, leukotrienes, purines, and 8 -isopentane ${ }^{140}$ that can be measured by mass spectrometry. ${ }^{141}$ The expression levels of inflammatory cytokines can be determined at the mRNA and protein levels in BA, or plasma ${ }^{142}$ as early predictors of lung disease. The expression of inflammatory cytokines in brushed nasal cells parallels that found in sputum. ${ }^{35,36}$ Thus, a decrease of tumor necrosis factor- $a$ and interleukin-8 in nasal cells mimicked that observed in patient's sputum and significantly correlated with the restoration of CFTR function during treatment with cysteamine and EGCG. ${ }^{35,36}$ Indeed, $88.4 \%$ of 'responders', defined as patients who restored CFTR function and mechanistic biomarkers of autophagy (such a BECN1 or SQSTM1) upon treatment reduced the levels of inflammatory cytokines by more than $30 \%{ }^{35,36}$

\section{Biomarkers of lung infection}

Finding appropriate biomarkers for the detection of pathogenic organisms in the lower respiratory tract, as bacterial cultures in BAL or measurements of the values of circulating anti-Pseudomonas antibodies, represent a challenging and debated issue. In addition, the analysis of exhaled breath volatile organic compounds is being investigated as a possible early biomarker of detection and eradications of pathogens in the lungs. ${ }^{143}$ The assessment of free neutrophil elastase can be used as a predictor of structural lung damage.

\section{Biomarkers of metabolic changes}

Metabolic changes have been observed in CF BAL and correlate with neutrophil counts and surrogate markers of lung function $\left(\mathrm{FEV}_{1}\right) \cdot{ }^{140,141}$ 
Table 2 Examples of clinical trials aiming at repairing CFTR protein function

\begin{tabular}{|c|c|c|c|}
\hline Age range & & CFTR mutation/function & Stage of development \\
\hline \multirow[t]{2}{*}{$\begin{array}{l}\mathrm{PTC} \\
\text { readthrough }\end{array}$} & $\begin{array}{l}\text { ATALUREN (PTC124) (PTC Therapeutics) } \\
\text { NCT00803205/NCT00351078 } \\
\text { NCT02107859 } \\
>6 y r s \text { and older }\end{array}$ & Non-sense mutations & Phase III \\
\hline & $\begin{array}{l}\text { ATALUREN (PTC124) } \\
\text { NCT02139306 } \\
\text { > 6yrs and older }\end{array}$ & $\begin{array}{l}\text { Non-sense mutations in patients not receiving } \\
\text { chronic inhaled aminoglycosides. }\end{array}$ & Phase III \\
\hline \multirow[t]{8}{*}{ Potentiators } & $\begin{array}{l}\text { IVACAFTOR }^{\mathrm{a}} \text { (Vertex Pharmaceuticals, Inc.) } \\
\text { NCT01705145 NCT00909532/NCT00909727 } \\
>2 \text { yrs and older }\end{array}$ & $\begin{array}{l}\text { G551D gating mutation and specific non-G551D } \\
\text { gating mutations at least on one allele }\end{array}$ & To patient \\
\hline & $\begin{array}{l}\text { NCT01614457 } \\
>6 y r s \text { and older }\end{array}$ & $\mathrm{R} 117 \mathrm{H}$ mutation at least on one allele & Phase III \\
\hline & $>18 y r s$ and older ${ }^{a}$ & $\mathrm{R} 117 \mathrm{H}$ mutation at least on one allele & To patient \\
\hline & $\begin{array}{l}\text { QBW251 (Novartis) } \\
\text { NCT02190604>18yrs }\end{array}$ & $\begin{array}{l}\text { Any mutation on one allele and class III, IV, V, VI } \\
\text { mutation on the other allele }\end{array}$ & Phase II \\
\hline & $\begin{array}{l}\text { GLPG1837 (Galapagos NV) } \\
\text { NCT02707562 } \\
\text { NCT02690519 }\end{array}$ & G551D gating mutation; S1251N mutation & Phase II \\
\hline & GLPG2451(Galapagos NV) & Multiple ascending oral doses in healthy & Phase I \\
\hline & $\begin{array}{l}\text { CTP-656 (Concert Pharmaceuticals) } \\
\text { NCT02599792 } \\
>18 y \text { rs }\end{array}$ & $\begin{array}{l}\text { Multiple ascending oral doses vs Ivacaftor and } \\
\text { placebo in healthy volunteers }\end{array}$ & Phase I \\
\hline & CP-628006 (Pfizer) & G551D gating mutation & Pre-clinical \\
\hline \multirow[t]{3}{*}{ Correctors } & $\begin{array}{l}\text { LUMACAFTOR (Vertex Pharmaceuticals) } \\
\text { NCT00865904 > 18yrs }\end{array}$ & F508del homozygous & Phase II \\
\hline & $\begin{array}{l}\text { FDL 160,FDL169,FDL042 (FDL282),FDL392, } \\
\text { FDL304 } \\
\text { Flatley Discovery Lab }\end{array}$ & CFTR Correctors & Pre-clinical \\
\hline & PYR-41 (Pfizer) & Decrease of CFTR ubiquitination & Pre-clinical \\
\hline \multirow[t]{16}{*}{$\begin{array}{l}\text { Correctors } \\
\text { +potentiators }\end{array}$} & $\begin{array}{l}\text { LUMACAFTOR+IVACAFTOR } \\
\text { NCT01931839/NCT01807923 }>12 \mathrm{yrs}^{\mathrm{a}} \text { and older }\end{array}$ & F508del homozygous & To patient \\
\hline & $\begin{array}{l}\text { NCT01897233 } \\
6-11 \text { yrs }\end{array}$ & F508del homozygous & Phase III \\
\hline & $\begin{array}{l}\text { NCT02797132 } \\
2-5 y r s\end{array}$ & F508del homozygous & Phase III \\
\hline & NCT01225211 > 18yrs & F508del on one allele & Phase II \\
\hline & $\begin{array}{l}\text { IVACAFTOR+VX-661 (Vertex Pharmaceuticals) } \\
\text { NCT02070744 > 18yrs }\end{array}$ & F508del homozygous & Phase II \\
\hline & NCT01531673 > 12yrs & $\begin{array}{l}\text { Homozygous or Heterozygous for the F508del- } \\
\text { CFTR mutation }\end{array}$ & Phase II \\
\hline & NCT02516410 > 12yrs & $\begin{array}{l}\text { F508del-CFTR on one allele and a second } \\
\text { mutation not likely to respond to VX-661 and/or } \\
\text { ivacaftor therapy }\end{array}$ & Phase III \\
\hline & NCT02412111>12yrs & $\begin{array}{l}\text { F508del-CFTR on one allele and a second gating } \\
\text { defect that is clinically demonstrated to be } \\
\text { Ivacaftor responsive }\end{array}$ & Phase III \\
\hline & NCT02508207 > 18yrs & Homozygous for the F508del-CFTR mutation & Phase II \\
\hline & NCT02392234 > 12yrs & Heterozygous for the F508del-CFTR mutation & Phase III \\
\hline & $\begin{array}{l}\text { NCT02953314 } \\
6 \text {-11yrs }\end{array}$ & $\begin{array}{l}\text { Homozygous or Heterozygous for the F508del- } \\
\text { CFTR mutation }\end{array}$ & Phase III \\
\hline & $\begin{array}{l}\text { VX-661+IVACAFTOR+VX-152 } \\
\text { NCT02951195> 18yrs }\end{array}$ & $\begin{array}{l}\text { Homozygous or Heterozygous for the F508del- } \\
\text { CFTR mutation }\end{array}$ & Phase II \\
\hline & $\begin{array}{l}\text { VX-661+IVACFTOR+VX-440 } \\
\text { NCT02951182>12yrs }\end{array}$ & & Phase II \\
\hline & $\begin{array}{l}\text { GLPG2222+GLPG2451 (Galapagos NV) } \\
\text { NCT02788721 }\end{array}$ & Multiple ascending doses in healthy volunteers & Phase I \\
\hline & $\begin{array}{l}\text { GLPG2222+GLPG2665+GLPG1837 } \\
\text { (Galapagos NV/Abbvie) }\end{array}$ & Multiple ascending doses in healthy volunteers & Phase I \\
\hline & $\begin{array}{l}\text { VX-371 (Vertex Pharmaceuticals,Inc., Parion) } \\
\text { NCT02709109>12yrs }\end{array}$ & $\begin{array}{l}\text { Homozygous for the F508del in treatment with } \\
\text { Orkambi }^{\text {Or. }}\end{array}$ & Phase II \\
\hline \multirow[t]{5}{*}{$\begin{array}{l}\text { Proteostasis } \\
\text { regulators }\end{array}$} & $\begin{array}{l}\text { CYSTEAMINE (Recordati) +EGCG }{ }^{\mathrm{C}} \\
\text { EudraCT no \#2013-001258-82 > 6yrs and older }\end{array}$ & $\begin{array}{l}\text { Class I, Class II on one allele and class II on the } \\
\text { other }\end{array}$ & Phase II \\
\hline & $\begin{array}{l}\text { N91115 (Nivalis Therapeutics,Inc.) } \\
\text { NCT0227593 > 18yrs }\end{array}$ & F508del homozygous & Phase I \\
\hline & $\begin{array}{l}\text { RIOCIGUAT (Bayer) } \\
\text { NCT02170025>18yrs }\end{array}$ & F508del homozygous & Phase II \\
\hline & SILDENAFIL & All CFTR mutations & Phase II \\
\hline & NCT0065952 $>14$ yrs & & \\
\hline Others & CAL Inhibitor CT007 (Calista Therapeutics) & CFTR stabilzer & Pre-clinical \\
\hline
\end{tabular}

${ }^{a} A$ pproved by regulatory agencies and licensed in different countries

${ }^{\mathrm{b}}$ Orkambi = Lumacaftor+lvacaftor

${ }^{c} E G C G=$ Epigallocathechin gallate 
soluble guanylate-cyclase stimulator riociguat (BAY63-2521, Bayer), which exhibits CFTR corrector activity, ${ }^{10}$ is being orally administered to F508del-CFTR homozygotes in a multicenter randomized phase 2 clinical trial (https://clinicaltrials.gov/) (NCT02170025). Similarly, the selective inhibitor of cGMPdependent phosphodiesterase-5, sildenafil, a repurposed drug, has been tested in a phase 2 study (NCT00659529) in homozygous adults F508del-CFTR for safety and efficacy $^{108}$ (Table 1). Two open-label clinical trials ${ }^{35,36}$ tested safety and efficacy of a combination of two proteostasis regulators, the repurposed drug cysteamine and the nutraceutical EGCG, that restore defective autophagy and inhibit CK2, respectively, in $\sim 40$ patients bearing F508del-CFTR or other class II CFTR mutations, either in homozygous or compound heterozygous form with class I mutations. ${ }^{36}$ In spite of the open-label study design and the small sample size, the treatment restored CFTR function (as measured both in sweat and nasal cells), that correlated with a decrease in pulmonary inflammation, and tended to improve $\mathrm{FEV}_{1}$ (Box 4, Table 1). The reported mucolytic and antibacterial effects of cysteamine ${ }^{109,110}$ (Box 1) might contribute to clinical benefit.

Heterogeneity in the therapeutic response rate characterizes CF and makes the interpretation of clinical trials problematic. There is general consensus that a personalized approach to treatment is required and that pre-selecting appropriate endpoints and biomarkers is crucial. ${ }^{94}$ Nonetheless, personalized approaches to treatment are still absent from most clinical trials that rather rely on the analysis of pooled data from large patient cohorts. The recent clinical trial with cysteamine and $\mathrm{EGCG}^{36}$ exemplifies the possibility to evaluate multiple biomarkers reflecting in vitro, ex vivo and in vivo responses on an individual, patient-by-patient basis (Box 4). Future clinical trials might be designed in a way that patients can be preselected for inclusion in the trial based on the reactivity of their cells to CFTR repair in vitro.

\section{Concluding Remarks}

The approval of Ivacaftor for clinical use in CF patients with gating mutations has been a major breakthrough, demonstrating that the development of etiological cures is an achievable goal. Nonetheless, it appears clear that the clinical utility of Ivacaftor is limited to a small fraction of $\sim 10 \%$ of CF patients, meaning that the largest segment of the CF population still awaits an etiological cure. Unfortunately, the most common CFTR mutant, F508del-CFTR is afflicted by a multifaceted defect that complicates drug discovery, likely because no single drug is able to accomplish the complete rescue of this mutant by a multipronged action. CFTR is at the center of an intricate network of regulatory systems, thus constituting the pivot of a wider landscape that amply transcends the mono-molecular vision of disease etiology. Strategies aiming at targeting the cellular environment perturbed by the CFTR defect have emerged. To date, these strategies are still considered alternative, and somehow antinomic, to CFTR modulators (correctors and potentiators) that are designed to specifically interact with the defective CFTR protein. However, accumulating evidence clearly indicates that targeting major systems of cellular proteostasis, including autophagy, the phosphoproteome and the chaperone machinery, can improve traffic and PM stability of F508del-CFTR in preclinical models and in patients. Thus, these apparently incompatible strategies may actually be complementary. Indeed, it can be hoped that variable combinations of CFTR modulators and proteostasis regulators will configure the authentic etiological CF therapy that can be adapted to each individual patient's needs. Future will tell whether such an etiological procedure for CF management will entail the expected reduction of symptomatic treatments, ultimately reducing the cost of CF treatment.

Beyond the obvious challenge to discover drugs (or drug combinations) that have an optimal effect on representative cell types afflicted by the F508del-CFTR mutation, it will be of the utmost importance to define an effective path leading from preclinical discovery to clinical implementation. Thus, a major conundrum in CF is the high individual variability in the response rate-even for patients sharing the same CFTR mutation-which obviously complicates the analysis of pooled data in clinical trials and increases the risk of failures in drug development. We believe that appropriate biomarkers will influence the decisions whether a given patient should be included in, and stay on, a trial, thereby improving the chances of successful CF drug development. Such biomarkers might include short-term cultures of patient-derived cells (such as respiratory epithelial cells or blood monocytes), followed by biochemical and functional measurements that directly assess CFTR expression and activity, respectively. Such measurements could be done on cells that are treated with the candidate drug in vitro (before the inclusion of the patient in the trial) or have been exposed to such drugs in vivo (after initiation of the trial, to evaluate their potential response). Alternatively, CFTR function may be directly measured in vivo, for instance by using the diagnostic sweat test. This biomarker-based procedure may lead to a more sophisticated patient stratification, beyond mere CFTR genotyping, thus improving the design of clinical trials and in fine truly personalizing CF therapy.

\section{Conflict of Interest}

LM, VR and GK are listed as inventors of a patent that protects the use of cysteamine for CF treatment.

Acknowledgements. GK is supported by the Ligue contre le Cancer (équipe labelisée); Agence National de la Recherche (ANR)_Projets blancs; ANR under the frame of E-Rare-2, the ERA-Net for Research on Rare Diseases; Association pour la recherche sur le cancer (ARC); Cancéropôle lle-de-France; Institut National du Cancer (INCa); Institut Universitaire de France; Fondation pour la Recherche Médicale (FRM); the European Commission (ArtForce); the European Research Council (ERC); the LeDucq Foundation; the LabEx Immuno-Oncology; the SIRIC Stratified Oncology Cell DNA Repair and Tumor Immune Elimination (SOCRATE); the SIRIC Cancer Research and Personalized Medicine (CARPEM); and the Paris Alliance of Cancer Research Institutes (PACRI). LM is supported by the E-Rare project 'RescueCFTRpreclinc'; the European Institute for Research in Cystic Fibrosis; VR is supported by Italian Ministry of Health, LL548; We thank Dr Valeria R. Villella, Senior Postdoc at the European Institute for Research in Cystic Fibrosis, for editing assistance. 


\section{Glossary}

Autophagy: A lysosomal pathway of degradation of cytoplasmic material that has previously been sequestered in autophagosomes.

Ubiquitin Proteasome Pathway (UPP): A complex mechanism for protein catabolism of unneeded or damaged proteins in the mammalian cytosol and nucleus by breaking peptide bonds, affectinga wide variety of cellular processes and substrates.

Proteostasis: A homeostatic system also termed proteostasis network that responds to multiple cell perturbations, bethey genetically determined or acquired.

High Throughput screening (HTS): An automated method that rapidly assesses the biological or chemical activity of a large numberof drug-like compounds.

Corrector: A small molecule that rescues the expression of misfolded CFTR (e.g., F508del) and allows its transport to the cell surface.

Potentiator: A small molecule that improves the ion transport activity of CFTR at the cell membrane.

Proteostasis regulator: A molecule that restores a functional proteostasis network. Nasal brushing: A method to obtain respiratory epithelial cells from the nasal cavities using a cotton swap.

Intestinal organoids: A novel method that allows long-term cultureof isolated intestinal crypts or intestinal stem cells.

Adeno-associated virus: A small virus that infects humans and some other primate species.

Nonsense mutation: A pointmutation in a sequence of DNA that results in a premature stop codon, or a nonsense codon, in the transcribed mRNA, and hence in a truncated, incomplete, and usually nonfunctional protein product.

Missense mutation: Change of a single base pair in DNA causing the substitution of one amino-acid residue by another one in the resulting protein.

Frameshift mutation: A mutation involving the insertion or deletion of a number of nucleotides that is not divisible by three.

Mouse model: A laboratory mouse used to study some aspects of human physiology or disease.

Exacerbation: An increase in severity of a condition usually used to imply a worsening of the state of the chest in CF.

Airway clearance techniques: Methods used to assist with secretion removal.

Sweat test: The diagnostic test for CF that measures the concentration of salt (sodium and chloride) in sweat.

Lung clearance index (LCI): Measure of lung physiology derived from multiple breath washout tests.

Mucociliary clearance of the respiratory tract: An important defense mechanism against inhaled pathogens.

Personalized medicine: Emerging practice of medicine that uses an individual's genetic profile to guide decisions made in regard to the prevention, diagnosis, and treatment of disease.

1. Riordan JR. Assembly of functional CFTR chloride channels. Annu Rev Physiol 2005; 67 : 701-718.

2. Cutting GR. Cystic fibrosis genetics: from molecular understanding to clinical application. Nat Rev Genet 2015; 16: 45-56.

3. Sanders DB, Fink AK. Background and epidemiology. Pediatr Clin North Am 2016; 63: 567-584.

4. Wang Y, Wrennall JA, Cai Z, Li H, Sheppard DN. Understanding how cystic fibrosis mutations disrupt CFTR function: from single molecules to animal models. Int $\mathrm{J}$ Biochem Cell Biol 2014; 52: 47-57.

5. Bozoky Z, Krzeminski M, Chong PA, Forman-Kay JD. Structural changes of CFTR R region upon phosphorylation: a plastic platform for intramolecular and intermolecular interactions. FEBS J 2013; 280: 4407-4416.

6. Gadsby DC, Vergani P, Csanady L. The ABC protein turned chloride channel whose failure causes cystic fibrosis. Nature 2006; 440: 477-483.

7. Corradi V, Vergani $P$, Tieleman DP. Cystic fibrosis transmembrane conductance regulator (CFTR):closed and open state channel models. J Biol Chem 2015; 290: 22891-22906.

8. Lewis HA, Wang C, Zhao X, Hamuro Y, Conners K, Kearins MC et al. Structure and dynamics of NBD1 from CFTR characterized using crystallography and hydrogen/ deuterium exchange mass spectrometry. J Mol Biol 2010; 396: 406-430.

9. Mendoza JL, Schmidt A, Li Q, Nuvaga E, Barrett T, Bridges RJ et al. Requirements for efficient correction of DeltaF508 CFTR revealed by analyses of evolved sequences. Cell 2012; 148: 164-174.

10. Quon BS, Rowe SM. New and emerging targeted therapies for cystic fibrosis. Bmj 2016; 352: i859.

11. De Boeck K, Amaral MD. Progress in therapies for cystic fibrosis. Lancet Respir Med 2016; 4: $662-674$.
12. Farinha $\mathrm{CM}$, Canato $\mathrm{S}$. From the endoplasmic reticulum to the plasma membrane: mechanisms of CFTR folding and trafficking. Cell Mol Life Sci 2017; 74: 39-55.

13. Okiyoneda T, Barriere H, Bagdany M, Rabeh WM, Du K, Hohfeld J et al. Peripheral protein quality control removes unfolded CFTR from the plasma membrane. Science 2010; 329 : 805-810.

14. Thibodeau PH, Richardson JM 3rd, Wang W, Millen L, Watson J, Mendoza JL et al. The cystic fibrosis-causing mutation deltaF508 affects multiple steps in cystic fibrosis transmembrane conductance regulator biogenesis. J Biol Chem 2010; 285: 35825-35835.

15. Sosnay PR, Siklosi KR, Van Goor F, Kaniecki K, Yu H, Sharma N et al. Defining the disease liability of variants in the cystic fibrosis transmembrane conductance regulator gene. Nat Genet 2013; 45: 1160-1167.

16. Van Goor F, Yu H, Burton B, Hoffman BJ. Effect of ivacaftor on CFTR forms with missense mutations associated with defects in protein processing or function. J Cyst Fibros 2014; 13: 29-36.

17. Veit G, Avramescu RG, Chiang AN, Houck SA, Cai Z, Peters KW et al. From CFTR biology toward combinatorial pharmacotherapy: expanded classification of cystic fibrosis mutations. Mol Biol Cell 2016; 27: 424-433.

18. Balch WE, Roth DM, Hutt DM. Emergent properties of proteostasis in managing cystic fibrosis. Cold Spring Harb Perspect Biol 2011; 3; doi: 10.1101/cshperspect.a004499.

19. Lopes-Pacheco M. CFTR modulators: shedding light on precision medicine for cystic fibrosis. Front Pharmacol 2016; 7: 275.

20. Pankow S, Bamberger C, Calzolari D, Martínez-Bartolomé S, Lavallée-Adam M, Balch WE et al. F508 CFTR interactome remodelling promotes rescue of cystic fibrosis. Nature 2015; 528: $510-516$.

21. Amaral MD, Balch WE. Hallmarks of therapeutic management of the cystic fibrosis functional landscape. J Cyst Fibros 2015; 14: 687-699.

22. Villella VR, Esposito S, Bruscia EM, Vicinanza M, Cenci S, Guido S et al. Disease-relevant proteostasis regulation of cystic fibrosis transmembrane conductance regulator. Cell Death Differ 2013; 20: 1101-1115.

23. Farinha CM, Matos P. Repairing the basic defect in cystic fibrosis-one approach is not enough. Febs j 2016; 283: 246-264.

24. Luciani A, Villella VR, Esposito S, Brunetti-Pierri N, Medina D, Settembre $C$ et al. Defective CFTR induces aggresome formation and lung inflammation in cystic fibrosis through ROSmediated autophagy inhibition. Nat Cell Biol 2010; 12: 863-875.

25. Luciani A, Villella VR, Esposito S, Gavina M, Russo I, Silano M et al. Targeting autophagy as a novel strategy for facilitating the therapeutic action of potentiators on DeltaF508 cystic fibrosis transmembrane conductance regulator. Autophagy 2012; 8: 1657-1672.

26. Villella VR, Esposito S, Bruscia EM, Maiuri MC, Raia V, Kroemer G et al. Targeting the Intracellular Environment in Cystic Fibrosis: Restoring Autophagy as a Novel Strategy to Circumvent the CFTR Defect. Front Pharmacol 2013; 4 : 1.

27. Abdulrahman BA, Khweek AA, Akhter A, Caution K, Kotrange S, Abdelaziz DH et al. Autophagy stimulation by rapamycin suppresses lung inflammation and infection by Burkholderia cenocepacia in a model of cystic fibrosis. Autophagy 2011; 7: 1359-1370.

28. Abdulrahman BA, Khweek AA, Akhter A, Caution K, Tazi M, Hassan H et al. Depletion of the ubiquitin-binding adaptor molecule SQSTM1/p62 from macrophages harboring cft DeltaF508 mutation improves the delivery of Burkholderia cenocepacia to the autophagic machinery. J Biol Chem 2013; 288: 2049-2058.

29. Junkins RD, McCormick C, Lin TJ. The emerging potential of autophagy-based therapies in the treatment of cystic fibrosis lung infections. Autophagy 2014; 10: 538-547.

30. Mayer ML, Blohmke CJ, Falsafi R, Fjell CD, Madera L, Turvey SE et al. Rescue of dysfunctional autophagy attenuates hyperinflammatory responses from cystic fibrosis cells. J Immunol 2013; 190: 1227-1238.

31. Luciani A, Villella VR, Vasaturo A, Giardino I, Raia V, Pettoello-Mantovani M et al. SUMOylation of tissue transglutaminase as link between oxidative stress and inflammation. $\mathrm{J}$ Immunol 2009; 183: 2775-2784.

32. Maiuri L, Luciani A, Giardino I, Raia V, Villella VR, D'Apolito M et al. Tissue transglutaminase activation modulates inflammation in cystic fibrosis via PPARgamma down-regulation. J Immunol 2008; 180: 7697-7705.

33. Farinha CM, Swiatecka-Urban A, Brautigan DL, Jordan P. Regulatory Crosstalk by Protein Kinases on CFTR Trafficking and Activity. Front Chem 2016; 4: 1.

34. Farinha $\mathrm{CM}$, Matos $\mathrm{P}$, Amaral MD. Control of cystic fibrosis transmembrane conductance regulator membrane trafficking: not just from the endoplasmic reticulum to the Golgi. FEBS J 2013; 280: 4396-4406.

35. De Stefano D, Villella VR, Esposito S, Tosco A, Sepe A, De Gregorio F et al. Restoration of CFTR function in patients with cystic fibrosis carrying the F508del-CFTR mutation. Autophagy 2014; 10: 2053-2074.

36. Tosco A, De Gregorio F, Esposito S, De Stefano D, Sana I, Ferrari E et al. A novel treatment of cystic fibrosis acting on-target: cysteamine plus epigallocatechin gallate for the autophagy-dependent rescue of class II-mutated CFTR. Cell Death Differ 2016; 23: 1380-1393.

37. Gill DR, Hyde SC. Delivery of genes into the CF airway. Thorax 2014; 69: 962-964.

38. Alton EW, Boyd AC, Porteous DJ, Davies G, Davies JC, Griesenbach U et al. A phase I/lla safety and efficacy study of nebulized liposome-mediated gene therapy for cystic fibrosis supports a multidose trial. Am J Respir Crit Care Med 2015; 192: 1389-1392.

39. Alton EW, Beekman JM, Boyd AC, Brand J, Carlon MS, Connolly MM. Preparation for a first-in-man lentivirus trial in patients with cystic fibrosis. Thorax 2017; 72: 137-147. 
40. Schwank G, Koo BK, Sasselli V, Dekkers JF, Heo I, Demircan T et al. Functional repair of CFTR by CRISPR/Cas9 in intestinal stem cell organoids of cystic fibrosis patients. Cell Stem Cell 2013; 13: 653-658.

41. Montiel-Gonzalez MF, Vallecillo-Viejo I, Yudowski GA, Rosenthal JJ. Correction of mutations within the cystic fibrosis transmembrane conductance regulator by site-directed RNA editing. Proc Natl Acad Sci USA 2013; 110: 18285-18290.

42. Xue X, Mutyam V, Tang L, Biswas S, Du M, Jackson LA et al. Synthetic aminoglycosides efficiently suppress cystic fibrosis transmembrane conductance regulator nonsense mutations and are enhanced by ivacaftor. Am J Respir Cell Mol Biol 2014; 50: 805-816.

43. Du M, Liu X, Welch EM, Hirawat S, Peltz SW, Bedwell DM. PTC124 is an orally bioavailable compound that promotes suppression of the human CFTR-G542X nonsense allele in a CF mouse model. Proc Natl Acad Sci USA 2008; 105: 2064-2069.

44. Oren YS, McClure ML, Rowe SM, Sorscher EJ, Bester AC, Manor M et al. The unfolded protein response affects readthrough of premature termination codons. EMBO Mol Med 2014; 6: 685-701.

45. Van Goor F, Hadida S, Grootenhuis PD, Burton B, Cao D, Neuberger T et al. Rescue of CF airway epithelial cell function in vitro by a CFTR potentiator, VX-770. Proc Natl Acad Sci USA 2009; 106: 18825-18830.

46. Ramsey BW, Davies J, McElvaney NG, Tullis E, Bell SC, Drevinek P et al. A CFTR potentiator in patients with cystic fibrosis and the G551D mutation. N Engl J Med 2011; 365 1663-1672.

47. De Boeck K, Munck A, Walker S, Faro A, Hiatt P, Gilmartin G et al. Efficacy and safety of ivacaftor in patients with cystic fibrosis and a non-G551D gating mutation. $J$ Cyst Fibros 2014; 13: 674-680.

48. Eckford PD, Li C, Ramjeesingh M, Bear CE. Cystic fibrosis transmembrane conductance regulator (CFTR) potentiator VX-770 (ivacaftor) opens the defective channel gate of mutant CFTR in a phosphorylation-dependent but ATP-independent manner. J Biol Chem 2012; 287: 36639-36649.

49. Yu H, Burton B, Huang CJ, Worley J, Cao D, Johnson JP Jr et al. Ivacaftor potentiation of multiple CFTR channels with gating mutations. J Cyst Fibros 2012; 11: 237-245.

50. Jih KY, Hwang TC. Vx-770 potentiates CFTR function by promoting decoupling between the gating cycle and ATP hydrolysis cycle. Proc Natl Acad Sci USA 2013; 110: 4404-4409.

51. Cholon DM, Quinney NL, Fulcher ML, Esther CR Jr., Das J, Dokholyan NV et al. Potentiator ivacaftor abrogates pharmacological correction of DeltaF508 CFTR in cystic fibrosis. Sci Transl Med 2014; 6: 246ra296.

52. Veit G, Avramescu RG, Perdomo D, Phuan PW, Bagdany M, Apaja PM et al. Some gating potentiators, including VX-770, diminish DeltaF508-CFTR functional expression. Sci Trans Med 2014; 6: 246ra297.

53. Boyle MP, Bell SC, Konstan MW, McColley SA, Rowe SM, Rietschel E et al. A CFTR corrector (lumacaftor) and a CFTR potentiator (ivacaftor) for treatment of patients with cystic fibrosis who have a phe508del CFTR mutation: a phase 2 randomised controlled trial. Lancet Respir Med 2014; 2: 527-538.

54. Wainwright CE, Elborn JS, Ramsey BW. Lumacaftor-Ivacaftor in Patients with Cystic Fibrosis Homozygous for Phe508del CFTR. N Engl J Med 2015; 373: 1783-1784.

55. Phuan PW, Veit G, Tan J, Roldan A, Finkbeiner WE, Lukacs GL et al. Synergy-based smallmolecule screen using a human lung epithelial cell line yields DeltaF508-CFTR correctors that augment VX-809 maximal efficacy. Mol Pharmacol 2014; 86: 42-51.

56. Lin WY, Sohma Y, Hwang TC. Synergistic potentiation of cystic fibrosis transmembrane conductance regulator gating by two chemically distinct potentiators, ivacaftor (VX-770) and 5-Nitro-2-(3-Phenylpropylamino) Benzoate. Mol Pharmacol 2016; 90: 275-285.

57. Pedemonte N, Lukacs GL, Du K, Caci E, Zegarra-Moran O, Galietta LJ et al. Smallmolecule correctors of defective DeltaF508-CFTR cellular processing identified by highthroughput screening. J Clin Invest 2005; 115: 2564-2571.

58. Kalid O, Mense M, Fischman S, Shitrit A, Bihler H, Ben-Zeev E et al. Small molecule correctors of F508del-CFTR discovered by structure-based virtual screening. J Comput Aided Mol Des 2010; 24: 971-991.

59. Van Goor F, Hadida S, Grootenhuis PD, Burton B, Stack JH, Straley KS et al. Correction of the F508del-CFTR protein processing defect in vitro by the investigational drug VX-809. Proc Natl Acad Sci USA 2011; 108: 18843-18848.

60. Okiyoneda T, Veit G, Dekkers JF, Bagdany M, Soya N, Xu H et al. Mechanism-based corrector combination restores DeltaF508-CFTR folding and function. Nat Chem Biol 2013 9: $444-454$.

61. Farinha CM, King-Underwood J, Sousa M, Correia AR, Henriques BJ, Roxo-Rosa M et al. Revertants, low temperature, and correctors reveal the mechanism of F508del-CFTR rescue by VX-809 and suggest multiple agents for full correction. Chem Biol 2013; 20 : 943-955.

62. Awatade NT, Uliyakina I, Farinha CM, Clarke LA, Mendes K, Sole A et al. Measurements of functional responses in human primary lung cells as a basis for personalized therapy for cystic fibrosis. EBioMedicine 2015; 2: 147-153.

63. Amaral MD. Novel personalized therapies for cystic fibrosis: treating the basic defect in all patients. J Intern Med 2015; 277: 155-166.

64. Pedemonte N, Tomati V, Sondo E, Caci E, Millo E, Armirotti A et al. Dual activity of aminoarylthiazoles on the trafficking and gating defects of the cystic fibrosis transmembrane conductance regulator chloride channel caused by cystic fibrosis mutations. J Biol Chem 2011; 286: 15215-15226.
65. Pesce E, Bellotti M, Liessi N, Guariento S, Damonte G, Cichero E et al. Synthesis and structure-activity relationship of aminoarylthiazole derivatives as correctors of the chloride transport defect in cystic fibrosis. Eur J Med Chem 2015; 99: 14-35.

66. Laselva O, Molinski S, Casavola V, Bear CE. The investigational Cystic Fibrosis drug Trimethylangelicin directly modulates CFTR by stabilizing the first membranespanning domain. Biochem Pharmacol 2016; 119: 85-92.

67. Lubamba B, Lecourt H, Lebacq J, Lebecque P, De Jonge H, Wallemacq P et al. Preclinical evidence that sildenafil and vardenafil activate chloride transport in cystic fibrosis. Am J Respir Crit Care Med 2008; 177: 506-515.

68. Hutt DM, Herman D, Rodrigues AP, Noel S, Pilewski JM, Matteson J et al. Reduced histone deacetylase 7 activity restores function to misfolded CFTR in cystic fibrosis. Nat Chem Biol 2010; 6: 25-33.

69. Wang X, Venable J, LaPointe P, Hutt DM, Koulov AV, Coppinger J et al. Hsp90 cochaperone Aha1 downregulation rescues misfolding of CFTR in cystic fibrosis. Cell 2006; 127: 803-815

70. Marozkina NV, Yemen S, Borowitz M, Liu L, Plapp M, Sun F et al. Hsp 70/Hsp 90 organizing protein as a nitrosylation target in cystic fibrosis therapy. Proc Natl Acad Sci USA 2010; 107: 11393-11398.

71. Zaman K, Sawczak V, Zaidi A, Butler M, Bennett D, Getsy P et al. Augmentation of CFTR maturation by S-nitrosoglutathione reductase. Am J Physiol Lung Cell Mol Physiol 2016; 310: L263-L270.

72. Du K, Karp PH, Ackerley C, Zabner J, Keshavjee S, Cutz E et al. Aggregates of mutant CFTR fragments in airway epithelial cells of CF lungs: new pathologic observations. J Cyst Fibros 2015; 14: 182-193.

73. Nurminskaya MV, Belkin AM. Cellular functions of tissue transglutaminase. Int Rev Cell Mol Biol 2012; 294: 1-97.

74. Galluzzi L, Bravo-San Pedro JM, Levine B, Green DR, Kroemer G. Pharmacological modulation of autophagy: therapeutic potential and persisting obstacles. Nat Rev Drug Discov. 2017; 16: 487-511.

75. Kroemer G, Marino G, Levine B. Autophagy and the integrated stress response. Mol Cell 2010; 40: 280-293.

76. Katsuragi $Y$, Ichimura $Y$, Komatsu M. p62/SQSTM1 functions as a signaling hub and an autophagy adaptor. Febs J 2015; 282: 4672-4678.

77. Marat AL, Haucke V. Phosphatidylinositol 3-phosphates-at the interface between cell signalling and membrane traffic. EMBO J 2016; 35: 561-579.

78. Romani L, Oikonomou V, Moretti S, lannitti RG, D'Adamo MC, Villella VR et al. Thymosin a1 represents a potential potent singlemolecule-based therapy for cystic fibrosis. Nat Med 2017; 5: 590-600

79. Billet $A$, Jia $Y$, Jensen $T$, Riordan JR, Hanrahan JW. Regulation of the cystic fibrosis transmembrane conductance regulator anion channel by tyrosine phosphorylation. FASEB J 2015; 29: 3945-3953.

80. Luz S, Cihil KM, Brautigan DL, Amaral MD, Farinha CM, Swiatecka-Urban A. LMTK2mediated phosphorylation regulates CFTR endocytosis in human airway epithelial cells. $J$ Biol Chem 2014; 289: 15080-15093.

81. Luz S, Kongsuphol P, Mendes Al, Romeiras F, Sousa M, Schreiber R et al. Contribution of casein kinase 2 and spleen tyrosine kinase to CFTR trafficking and protein kinase A-induced activity. Mol Cell Biol 2011; 31: 4392-4404.

82. Pagano MA, Marin O, Cozza G, Sarno S, Meggio F, Treharne KJ et al. Cystic fibrosis transmembrane regulator fragments with the Phe508 deletion exert a dual allosteric control over the master kinase CK2. Biochem J 2010; 426: 19-29.

83. V Venerando A, Ruzzene M, Pinna LA. Casein kinase: the triple meaning of a misnomer. Biochem J 2014; 460: 141-156.

84. Pedemonte N, Tomati V, Sondo E, Galietta LJ. Influence of cell background on pharmacological rescue of mutant CFTR. Am J Physiol Cell Physiol 2010; 298 : C866-C874.

85. McDougall CM, Blaylock MG, Douglas JG, Brooker RJ, Helms PJ, Walsh GM. Nasal epithelial cells as surrogates for bronchial epithelial cells in airway inflammation studies. Am J Respir Cell Mol Biol 2008; 39: 560-568.

86. Dekkers JF, Wiegerinck CL, de Jonge HR, Bronsveld I, Janssens HM, de Winter-de Groot $\mathrm{KM}$ et al. A functional CFTR assay using primary cystic fibrosis intestinal organoids. Nat Med 2013; 19: 939-945.

87. Tosoni K, Cassidy D, Kerr B, Land SC, Mehta A. Using drugs to probe the variability of trans-epithelial airway resistance. PLOS ONE 2016; 11: e0149550.

88. Sorio C, Buffelli M, Angiari C, Ettorre M, Johansson J, Vezzalini M et al. Defective CFTR expression and function are detectable in blood monocytes: development of a new blood test for cystic fibrosis. PLOS ONE 2011; 6: e22212,..

89. Lavelle GM, White MM, Browne N, McElvaney NG, Reeves EP. Animal models of cystic fibrosis pathology: phenotypic parallels and divergences. Biomed Res Int 2016: 5258727.

90. Darrah RJ, Mitchell AL, Campanaro CK, Barbato ES, Litman P, Sattar A et al. Early pulmonary disease manifestations in cystic fibrosis mice. J Cyst Fibros 2016; 15: 736-744.

91. Bruscia EM, Zhang PX, Barone C, Scholte BJ, Homer R, Krause DS et al. Increased susceptibility of Cftr-/- mice to LPS-induced lung remodeling. Am J Physiol Lung Cell Mol Physiol 2016; 310: L711-L719.

92. Rogers CS, Stoltz DA, Meyerholz DK, Ostedgaard LS, Rokhlina T, Taft PJ et al. Disruption of the CFTR gene produces a model of cystic fibrosis in newborn pigs. Science 2008; 321 : $1837-1841$ 
93. Sun X, Sui H, Fisher JT, Yan Z, Liu X, Cho HJ et al. Disease phenotype of a ferret CFTRknockout model of cystic fibrosis. J Clin Invest 2010; 120: 3149-3160.

94. De Boeck K, Kent L, Davies J, Derichs N, Amaral M, Rowe SM et al. CFTR biomarkers: time for promotion to surrogate end-point. Eur Respir J 2013; 41: 203-216.

95. Kent L, Reix P, Innes JA, Zielen S, Le Bourgeois M, Braggion C et al. Lung clearance index: evidence for use in clinical trials in cystic fibrosis. J Cyst Fibros 2014; 13: 123-138.

96. Taylor-Robinson D, Whitehead M, Diderichsen F, Olesen HV, Pressler T, Smyth RL et al. Understanding the natural progression in \%FEV1 decline in patients with cystic fibrosis: a longitudinal study. Thorax 2012; 67: 860-866.

97. Kerem E, Konstan MW, De Boeck K, Accurso FJ, Sermet-Gaudelus I, Wilschanski M et al. Ataluren for the treatment of nonsense-mutation cystic fibrosis: a randomised, double-blind, placebo-controlled phase 3 trial. Lancet Respir Med 2014; 2: 539-547.

98. Aslam A, Jahnke N, Remmington T, Southern KW. Ataluren and similar compounds (specific therapies for premature terminationcodon class I mutations) for cystic fibrosis. Paediatr Respir Rev2017 Epub ahead of print Apr 27 2017; doi:10.1016/.jprrv.2017.04.001.

99. Davies JC, Wainwright CE, Canny GJ, Chilvers MA, Howenstine MS, Munck A et al. Efficacy and safety of ivacaftor in patients aged 6 to 11 years with cystic fibrosis with a G551D mutation. Am J Respir Crit Care Med 2013; 187: 1219-1225.

100. McKone EF, Borowitz D, Drevinek P, Griese M, Konstan MW, Wainwright C et al. Longterm safety and efficacy of ivacaftor in patients with cystic fibrosis who have the Gly551AspCFTR mutation: a phase 3, open-label extension study (PERSIST). Lancet Respir Med 2014: 2: 902-910.

101. Davies JC, Cunningham S, Harris WT, Lapey A, Regelmann WE, Sawicki GS et al. Safety, pharmacokinetics, and pharmacodynamics of ivacaftor in patients aged 2-5 years with cystic fibrosis and a CFTR gating mutation (KIWI): an open-label, single-arm study. Lancet Respir Med 2016; 4: 107-115.

102. Sawicki GS, McKone EF, Pasta DJ, Millar SJ, Wagener JS, Johnson CA et al. Sustained Benefit from ivacaftor demonstrated by combining clinical trial and cystic fibrosis patient registry data. Am J Respir Crit Care Med 2015; 192: 836-842.

103. Ferkol T, Quinton P. Precision Medicine: At What Price?. Am J Respir Crit Care Med 2015; 192: 658-659.

104. Clancy JP, Rowe SM, Accurso FJ, Aitken ML, Amin RS, Ashlock MA et al. Results of a phase lla study of VX-809, an investigational CFTR corrector compound, in subjects with cystic fibrosis homozygous for the F508del-CFTR mutation. Thorax 2012; 67: 12-18.

105. Elborn JS, Ramsey BW, Boyle MP, Konstan MW, Huang X, Marigowda G et al. Efficacy and safety of lumacaftor/ivacaftor combination therapy in patients with cystic fibrosis homozygous for Phe508del CFTR by pulmonary function subgroup: a pooled analysis. Lancet Respir Med 2016; 4: 617-626.

106. Yang C, Chilvers M, Montgomery M, Nolan SJ. Dornase alfa for cystic fibrosis. Cochrane Database Syst Rev 2016; 4: Cd001127.

107. Konstan MW, McKone EF, Moss RB, Marigowda G, Tian S, Waltz D et al. Assessment of safety and efficacy of long-term treatment with combination lumacaftor and ivacaftor therapy in patients with cystic fibrosis homozygous for the F508del-CFTR mutation (PROGRESS): a phase 3, extension study. Lancet Respir Med 2017; 5: 107-118.

108. Taylor-Cousar JL, Wiley C, Felton LA St, Clair C, Jones M, Curran-Everett D et al. Pharmacokinetics and tolerability of oral sildenafil in adults with cystic fibrosis lung disease. J Cyst Fibros 2015; 14: 228-236.

109. Charrier C, Rodger C, Robertson J, Kowalczuk A, Shand N, Fraser-Pitt D et al. Cysteamine (Lynovex $(\mathrm{R}))$, a novel mucoactive antimicrobial \& antibiofilm agent for the treatment of cystic fibrosis. Orphanet J Rare Dis 2014; 9: 189.

110. Devereux G, Fraser-Pitt D, Robertson J, Devlin E, Mercer D, O'Neil D. Cysteamine as a future intervention in cystic fibrosis against current and emerging pathogens: a patientbased ex vivo study confirming its antimicrobial and mucoactive potential in sputum. EBioMedicine 2015; 2: 1507-1512.

111. Smyth AR, Bell SC, Bojcin S, Bryon M, Duff A, Flume P et al. European cystic fibrosis society standards of care: Best Practice guidelines. J Cyst Fibros 2014; 13(Suppl 1): S23-S42.

112. Oermann CM, Retsch-Bogart GZ, Quittner AL, Gibson RL, McCoy KS, Montgomery AB et al. An 18-month study of the safety and efficacy of repeated courses of inhaled aztreonam Iysine in cystic fibrosis. Pediatr Pulmonol 2010; 45: 1121-1134.

113. Konstan MW, Flume PA, Kappler M, Chiron R, Higgins M, Brockhaus F et al. Safety, efficacy and convenience of tobramycin inhalation powder in cystic fibrosis patients: The EAGER trial. J Cyst Fibros 2011; 10: 54-61.

114. Schuster A, Haliburn C, Doring G, Goldman MH. Safety efficacy and convenience of colistimethate sodium dry powder for inhalation (Colobreathe DPI) in patients with cystic fibrosis: a randomised study. Thorax 2013; 68: 344-350.

115. Clancy JP, Dupont L, Konstan MW, Billings J, Fustik S, Goss CH et al. Phase II studies of nebulised Arikace in CF patients with Pseudomonas aeruginosa infection. Thorax 2013; 68 : 818-825.

116. Geller DE, Flume PA, Griffith DC, Morgan E, White D, Loutit JS et al. Pharmacokinetics and safety of MP-376 (levofloxacin inhalation solution) in cystic fibrosis subjects. Antimicrob Agents Chemother 2011; 55: 2636-2640.

117. Trapnell BC, McColley SA, Kissner DG, Rolfe MW, Rosen JM, McKevitt M et al. Fosfomycin/ tobramycin for inhalation in patients with cystic fibrosis with pseudomonas airway infection. Am J Respir Crit Care Med 2012; 185: 171-178.
118. Lashua LP, Melvin JA, Deslouches B, Pilewski JM, Montelaro RC, Bomberger JM Engineered cationic antimicrobial peptide (eCAP) prevents Pseudomonas aeruginosa biofilm growth on airway epithelial cells. J Antimicrob Chemother 2016; 71: 2200-2207.

119. Elkins MR, Robinson M, Rose BR, Harbour C, Moriarty CP, Marks GB et al. A controlled trial of long-term inhaled hypertonic saline in patients with cystic fibrosis. N Engl J Med 2006; 354: 229-240.

120. Bilton D, Daviskas E, Anderson SD, Kolbe J, King G, Stirling RG et al. Phase 3 randomized study of the efficacy and safety of inhaled dry powder mannitol for the symptomatic treatment of non-cystic fibrosis bronchiectasis. Chest 2013; 144: 215-225.

121. Bucki R, Cruz K, Pogoda K, Eggert A, Chin L, Ferrin M et al. Enhancement of Pulmozyme activity in purulent sputum by combination with poly-aspartic acid or gelsolin. $J$ Cyst Fibros 2015; 14: 587-593.

122. Konstan MW, Wagener JS, Pasta DJ, Millar SJ, Jacobs JR, Yegin A et al. Clinical use of dornase alpha is associated with a slower rate of FEV1 decline in cystic fibrosis. Pediatr Pulmonol 2011; 46: 545-553.

123. Pons G, Marchand MC, d'Athis $P$, Sauvage E, Foucard C, Chaumet-Riffaud $P$ et al. French multicenter randomized double-blind placebo-controlled trial on nebulized amiloride in cystic fibrosis patients. The Amiloride-AFLM Collaborative Study Group. Pediatr Pulmonol 2000; 30: 25-31.

124. Clark KL, Hughes SA, Bulsara P, Coates J, Moores K, Parry J et al. Pharmacological characterization of a novel ENaCalpha siRNA (GSK2225745) with potential for the treatment of cystic fibrosis. Mol Ther Nucleic Acids 2013; 2: e65.

125. Mall MA, Galietta LJ. Targeting ion channels in cystic fibrosis. J Cyst Fibros 2015; 14 561-570.

126. Tang XX, Ostedgaard LS, Hoegger MJ, Moninger TO, Karp PH, McMenimen JD et al. Acidic pH increases airway surface liquid viscosity in cystic fibrosis. J Clin Invest 2016; 126: 879-891.

127. Abou Alaiwa MH, Launspach JL, Sheets KA, Rivera JA, Gansemer ND, Taft PJ et al. Repurposing tromethamine as inhaled therapy to treat CF airway disease. JCl Insight 2016; 1: $56-59$.

128. Okiyoneda T, Lukacs GL. Fixing cystic fibrosis by correcting CFTR domain assembly. J Cell Biol 2012; 199: 199-204.

129. Rabeh WM, Bossard F, Xu H, Okiyoneda T, Bagdany M, Mulvihill CM et al. Correction of both NBD1 energetics and domain interface is required to restore DeltaF508 CFTR folding and function. Cell 2012; 148: 150-163.

130. Bali V, Lazrak A, Guroji P, Matalon S, Bebok Z. Mechanistic approaches to improve correction of the most common disease-causing mutation in cystic fibrosis. PLOS ONE 2016; 11: e0155882.

131. Wilke M, Buijs-Offerman RM, Aarbiou J, Colledge WH, Sheppard DN, Touqui L et al. Mouse models of cystic fibrosis: phenotypic analysis and research applications. J Cyst Fibros 2011; 10(Suppl 2): S152-S171.

132. Legssyer R, Huaux F, Lebacq J, Delos M, Marbaix E, Lebecque P et al. Azithromycin reduces spontaneous and induced inflammation in DeltaF508 cystic fibrosis mice. Respir Res 2006; 7: 134-137.

133. van Heeckeren AM, Tscheikuna J, Walenga RW, Konstan MW, Davis PB, Erokwu B et al. Effect of Pseudomonas infection on weight loss, lung mechanics, and cytokines in mice. Am J Respir Crit Care Med 2000; 161: 271-279.

134. Robert R, Carlile GW, Liao J, Balghi H, Lesimple P, Liu N et al. Correction of the Delta phe508 cystic fibrosis transmembrane conductance regulator trafficking defect by the bioavailable compound glafenine. Mol Pharmacol 2010; 77: 922-930.

135. Noel S, Wilke M, Bot AG, De Jonge HR, Becq F. Parallel improvement of sodium and chloride transport defects by miglustat (n-butyldeoxynojyrimicin) in cystic fibrosis epithelial cells. J Pharmacol Exp Ther 2008; 325: 1016-1023.

136. Mall M, Grubb BR, Harkema JR, O'Neal WK, Boucher RC. Increased airway epithelial Na+ absorption produces cystic fibrosis-like lung disease in mice. Nat Med 2004; 10: 487-493.

137. Accurso FJ, Van Goor F, Zha J, Stone AJ, Dong Q, Ordonez CL et al. Sweat chloride as a biomarker of CFTR activity: proof of concept and ivacaftor clinical trial data. J Cyst Fibros 2014; 13: 139-147.

138. Clancy JP, Szczesniak RD, Ashlock MA, Ernst SE, Fan L, Hornick DB et al. Multicenter intestinal current measurements in rectal biopsies from CF and non-CF subjects to monitor CFTR function. PLoS One 2013; 8: e73905.

139. Liou TG, Adler FR, Keogh RH, Li Y, Jensen JL, Walsh W et al. Sputum biomarkers and the prediction of clinical outcomes in patients with cystic fibrosis. PLOS ONE 2012; 7: e42748.

140. Patel K, Davis SD, Johnson R, Esther CR Jr. Exhaled breath condensate purines correlate with lung function in infants and preschoolers. Pediatr Pulmonol 2013; 48: 182-187.

141. Esther CR Jr, Coakley RD, Henderson AG, Zhou YH, Wright FA, Boucher RC. Metabolomic Evaluation of Neutrophilic Airway Inflammation in Cystic Fibrosis. Chest 2015; 148 : 507-515.

142. Harris WT, Muhlebach MS, Oster RA, Knowles MR, Clancy JP, Noah TL. Plasma TGF-beta (1) in pediatric cystic fibrosis: potential biomarker of lung disease and response to therapy. Pediatr Pulmonol 2011; 46: 688-695.

143. Thorn RM, Reynolds DM, Greenman J. Multivariate analysis of bacterial volatile compound profiles for discrimination between selected species and strains in vitro. $J$ Microbiol Methods 2011; 84: 258-264.

144. Meacham GC, Patterson C, Zhang W, Younger JM, Cyr DM. The Hsc70 co-chaperone CHIP targets immature CFTR for proteasomal degradation. Nat Cell Biol 2001; 3: 100-105. 
145. Hutt DM, Roth DM, Chalfant MA, Youker RT, Matteson J, Brodsky JL et al. FK506 binding protein 8 peptidylprolyl isomerase activity manages a late stage of cystic fibrosis transmembrane conductance regulator (CFTR) folding and stability. J Biol Chem 2012; 287 21914-21925.

146. Michelsen K, Yuan H, Schwappach B. Hide and run. Arginine-based endoplasmicreticulum-sorting motifs in the assembly of heteromultimeric membrane proteins. EMBO Rep 2005; 6: 717-722.

147. Wang X, Matteson J, An Y, Moyer B, Yoo JS, Bannykh S et al. COPII-dependent export of cystic fibrosis transmembrane conductance regulator from the ER uses a di-acidic exit code. J Cell Biol 2004; 167: 65-74.

148. Yoo JS, Moyer BD, Bannykh S, Yoo HM, Riordan JR, Balch WE. Non-conventiona trafficking of the cystic fibrosis transmembrane conductance regulator through the early secretory pathway. J Biol Chem 2002; 277: 11401-11409.

149. Gee HY, Noh SH, Tang BL, Kim KH, Lee MG. Rescue of DeltaF508-CFTR trafficking via a GRASP-dependent unconventional secretion pathway. Cell 2011; 146: 746-760.

150. Mendes F, Farinha CM, Felicio V, Alves PC, Vieira I, Amaral MD. BAG-1 stabilizes mutant F508del-CFTR in a ubiquitin-like-domain-dependent manner. Cell Physiol Biochem 2012; 30: 1120-1133

151. Bomberger JM, Barnaby RL, Stanton BA. The deubiquitinating enzyme USP10 regulates the post-endocytic sorting of cystic fibrosis transmembrane conductance regulator in airway epithelial cells. J Biol Chem 2009; 284: 18778-18789.

152. Gentzsch M, Chang XB, Cui L, Wu Y, Ozols VV, Choudhury A et al. Endocytic trafficking routes of wild type and DeltaF508 cystic fibrosis transmembrane conductance regulator. Mol Biol Cell 2004; 15: 2684-2696.

153. Swiatecka-Urban A, Brown A, Moreau-Marquis S, Renuka J, Coutermarsh B, Barnaby R et al. The short apical membrane half-life of rescued \{Delta\}F508-cystic fibrosis transmembrane conductance regulator (CFTR) results from accelerated endocytosis of \{Delta\}F508-CFTR in polarized human airway epithelial cells. J Biol Chem 2005; 280 : 36762-36772.

154. Okiyoneda T, Apaja PM, Lukacs GL. Protein quality control at the plasma membrane. Curr Opin Cell Biol 2011; 23: 483-491.

155. Silvis MR, Bertrand CA, Ameen N, Golin-Bisello F, Butterworth MB, Frizzell RA et al. Rab11b regulates the apical recycling of the cystic fibrosis transmembrane conductance regulator in polarized intestinal epithelial cells. Mol Biol Cell 2009; 20: 2337-2350.

156. Haggie PM, Kim JK, Lukacs GL, Verkman AS. Tracking of quantum dot-labeled CFTR shows near immobilization by C-terminal PDZ interactions. Mol Biol Cell 2006; 17 4937-4945.

157. Lobo MJ, Amaral MD, Zaccolo M, Farinha CM. EPAC1 activation by cAMP stabilizes CFTR at the membrane by promoting its interaction with NHERF1. J Cell Sci 2016; 129 : 2599-2612.

158. Faria D, Dahimene S, Alessio L, Scott-Ward T, Schreiber R, Kunzelmann K et al. Effect of Annexin A5 on CFTR: regulated traffic or scaffolding? Mol Membr Biol 2011; 28: 14-29.

159. Castellani S, Guerra L, Favia M, Di Gioia S, Casavola V, Conese M. NHERF1 and CFTR restore tight junction organisation and function in cystic fibrosis airway epithelial cells: role of ezrin and the RhoA/ROCK pathway. Lab Invest 2012; 92: 1527-1540.

160. Colas J, Faure G, Saussereau E, Trudel S, Rabeh WM, Bitam S et al. Disruption of cytokeratin-8 interaction with F508del-CFTR corrects its functional defect. Hum Mol Genet 2012; 21: 623-634.

161. Moniz M, Sousa S, Moraes M, Mendes BJ, Palma Al, Barreto M. C, et al. HGF stimulation of Rac1 signaling enhances pharmacological correction of the most prevalent cystic fibrosis mutant F508del-CFTR. ACS Chem Biol 2013; 8: 432-442.

162. Thoresen SB, Pedersen NM, Liestol K, Stenmark H. A phosphatidylinositol 3-kinase class III sub-complex containing VPS15, VPS34, Beclin 1, UVRAG and BIF-1 regulates cytokinesis and degradative endocytic traffic. Exp Cell Res 2010; 316: 3368-3378.

163. Conrad C, Lymp J, Thompson V, Dunn C, Davies Z, Chatfield B et al. Long-term treatment with oral $\mathrm{N}$-acetylcysteine: affects lung function but not sputum inflammation in cystic fibrosis subjects. A phase II randomized placebo-controlled trial. J Cyst Fibros 2015; 14 219-227.
164. Ratjen F, Durham T, Navratil T, Schaberg A, Accurso FJ, Wainwright $\mathrm{C}$ et al. Long term effects of denufosol tetrasodium in patients with cystic fibrosis. J Cyst Fibros 2012; 11: 539-549.

165. Fellner RC, Terryah ST, Tarran R. Inhaled protein/peptide-based therapies for respiratory disease. Mol Cell Pediatr 2016; 3: 16.

166. Cheng K, Ashby D, Smyth RL. Oral steroids for long-term use in cystic fibrosis. Cochrane Database Syst Rev 2015: Cd00040710.1002/14651858.CD000407.pub4.

167. Balfour-Lynn IM, Welch K. Inhaled corticosteroids for cystic fibrosis. Cochrane Database Syst Rev 2016: Cd00191510.1002/14651858.CD001915.pub5.

168. Konstan MW, Doring G, Heltshe SL, Lands LC, Hilliard KA, Koker P et al. A randomized double blind, placebo controlled phase 2 trial of BIIL 284 BS (an LTB4 receptor antagonist) for the treatment of lung disease in children and adults with cystic fibrosis. J Cyst Fibros 2014; 13: 148-155.

169. Lands LC, Stanojevic S. Oral non-steroidal anti-inflammatory drug therapy for lung disease in cystic fibrosis. Cochrane Database Syst Rev 2016; 4: Cd001505.

170. Carlile GW, Robert R, Goepp J, Matthes E, Liao J, Kus B et al. Ibuprofen rescues mutant cystic fibrosis transmembrane conductance regulator trafficking. J Cyst Fibros 2015; 14: $16-25$.

171. Saiman L, Anstead M, Mayer-Hamblett N, Lands LC, Kloster M, Hocevar-Trnka J et al. Effect of azithromycin on pulmonary function in patients with cystic fibrosis uninfected with Pseudomonas aeruginosa: a randomized controlled trial. Jama 2010; 303: 1707-1715.

172. Griese M, Latzin P, Kappler M, Weckerle K, Heinzlmaier T, Bernhardt T et al. alpha1Antitrypsin inhalation reduces airway inflammation in cystic fibrosis patients. Eur Respir $J$ 2007; 29: 240-250.

173. Visca A, Bishop CT, Hilton S, Hudson VM. Oral reduced L-glutathione improves growth in pediatric cystic fibrosis patients. J Pediatr Gastroenterol Nutr 2015; 60: 802-810.

174. Calabrese C, Tosco A, Abete P, Carnovale V, Basile C, Magliocca A et al. Randomized, single blind, controlled trial of inhaled glutathione vs placebo in patients with cystic fibrosis. $J$ Cyst Fibros 2015; 14: 203-210.

175. Moss RB, Mayer-Hamblett N, Wagener J, Daines C, Hale K, Ahrens R et al. Randomized, double-blind, placebo-controlled, dose-escalating study of aerosolized interferon gamma$1 \mathrm{~b}$ in patients with mild to moderate cystic fibrosis lung disease. Pediatr Pulmonol 2005; 39: 209-218.

176. Blau HAM, Mussaffi $\mathrm{H}$ et al. in 28th Annual North American Cystic Fibrosis Conference; 2014; 226. (Atlanta, GA, USA, Oct 9-11, 2014. 226.).

177. Grasemann $\mathrm{H}$, Tullis $\mathrm{E}$, Ratjen $\mathrm{F}$. A randomized controlled trial of inhaled L-arginine in patients with cystic fibrosis. J Cyst Fibros 2013; 12: 468-474.

178. Hraiech S, Bregeon F, Rolain JM. -based therapy in cystic fibrosis-associated Pseudomonas aeruginosa infections: rationale and current status. Drug Des Devel Ther 2015; 9: 3653-3663.

179. Waters EM, Neill DR, Kaman B, Sahota JS, Clokie MRJ, Winstanley C et al. Phage therapy is highly effective against chronic lung infections with Pseudomonas aeruginosa. Thorax 2017; 72: 666-667.

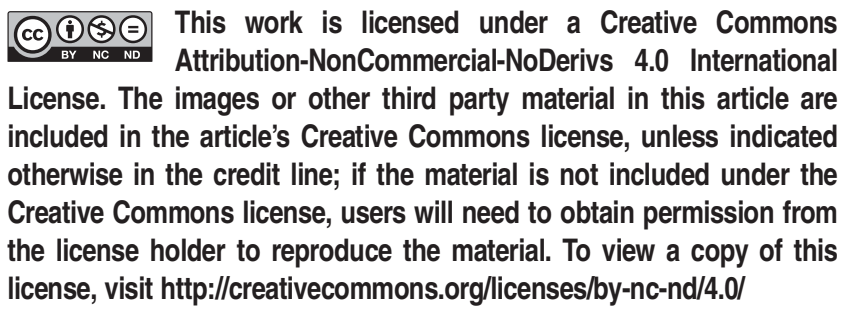

(C) The Author(s) 2017 